\title{
Lifestyle Modification among Post Renal Transplant Recipients
}

\author{
${ }^{1}$ Shimaa Hassan El said, ${ }^{2}$ Dr.Samar El- Hoseiny Abd El-Raouf,${ }^{3}$ Dr .Khald \\ Farook El -Dahashan, ${ }^{4}$ Dr Rabab Gad, \\ Abd El-Kader ${ }^{\text {I} B . S c, ~ F a c u l t y ~ O f ~ N u r s i n g, ~ M a n s o u r a ~ U n i v e r s i t y ' ~}$ \\ ${ }^{2}$ Associated Professor Of Community Health Nursing, Faculty Of Nursing, Mansoura University, \\ ${ }^{3}$ Associated Professor Of Nephrology Medicine, Faculty Of Medicine Mansoura University, \\ ${ }^{4}$ Lecturer Of Community Health Nursing, Faculty Of Nursing, Mansoura University, Egypt
}

\begin{abstract}
Background: The long term success of renal transplantation depends on many factors, follow a healthy lifestyle including proper diet, exercise, and weight control, beside that a good adherence toward immunosuppressive medication, the same as compliance toward recommended behaviors and healthy advices. A cross sectional study aimed to assess the lifestyle modification among renal transplant recipients. A simple random sample to recruit 120 renal transplant recipients from the record belonged to post renal transplanted recipients at Nephrology and Urology Center affiliated to Mansura University. Data were collected through five tools: 1) structure interview to assess socio demographic and economic characteristics, 2) post renal transplant recipients' health profile including a) medical history, b) physical examination, $c$ ) signs and symptoms related to side effects of immunosuppressive medications, and d) laboratory investigations, 3) structured interview to assess renal transplant recipients' lifestyle modification, 4) structure interview to assess post renal transplant recipients' medications adherence, and 5) structure interview to assess renal transplant recipients' compliance. The results of the present study illustrated that more than two thirds and more than half of renal transplant recipients were males and married respectively, the majority of them were overweight (more than $30 \mathrm{Kg} / \mathrm{m} 2$ ), the majority of renal transplant recipients had fried food, and the most of them used animal oil, more than three fourths of renal transplant recipients drank more than 2.5 liters of water per day and two thirds of them had 1.5 -3 L urine per day, only $12.5 \%$ of the renal transplant recipients were cigarette smoker, the majority of them (80.0\%) had a good adherence to immunosuppressive medication post renal transplantation, the majority $(87.5 \%)$ of renal transplant recipients were partially compliant, there were statistically significant association between sex, marital status and occupation of renal transplant recipients and their total adherence score to immunosuppressive medication $p=0.023,0.005$ and 0.046 respectively, there were statistically significant association between age, marital status, occupation and residence of renal transplant recipients and their total score compliance $p=0.026,0.00,0.288$ and 0.015 respectively, and finally there were a significant correlation between renal transplant recipients, adherence and their compliance, $(r=+0.272 \& p=0.003)$. The study recommended that periodical assessment for lifestyle, adherence to immunosuppressive medication and compliance to therapeutic regimen of post renal transplant recipient, and develops and implements an educational program for renal transplant recipients concerning lifestyle modification post transplantation.
\end{abstract}

\section{Introduction}

Renal transplantation (RTx) is the organ transplant of a healthy kidney to patient with end-stage kidney disease (ESKD), renal transplantation is typically classified as deceased donor (formally known as cadaveric) or living donor transplantation depend on the source of the donor organ. Living donor renal transplants are further characterized as genetically related (living-related) or non-related (living-unrelated) transplants, it depending on whether a biological relationship exists between the donor and recipient (Morrise $\boldsymbol{\&}$ Stuart, 2008).

Renal transplantation has become a progressive and novelty field, the number of renal transplant recipients continues to increase (Squifflet, 2011). Renal transplantation offer the best chance of long term survival and complete restoration and it is the most cost effective treatment in the patient with End Stage Renal Disease (ESRD). It can restore normal kidney function and correct all metabolic abnormalities of chronic kidney disease (Walker, Ralston \& Colledge, 2011).

A successful RTx consistently report a better quality of life than patient suffering hemodialysis or peritoneal dialysis (Danovitch, 2010). The prognosis post renal transplantation has improved. Recent UK statistics for renal transplantation stated that $96 \%$ patient survival and $92 \%$ graft survival at first year, $87 \%$ patient survival, and $82 \%$ graft survival at the first five years (Walker, Ralston \& Colledge, 2011). National Kidney Foundation (NKF, 2013) stated that there are 16,812 renal transplants had taken place in US and 11,043 came from deceased donor and 5,769 came from living donor. In Egypt living donor is only the legal choice for renal transplantation and 1,200 recipients using live donor are carried out every year where the incidence of ESRD is 
200 people in every million (El-Agrody, 2007). Urology and Nephrology center at Mansoura reported that total number of renal transplantation was 2795 from year 1976 till 2016 with an average of 100 transplant recipients annually. The rate of transplantation is 32 per 1000 dialysis patient per year that is much lower than North Europe 135 per 1000 dialysis patient (Gomaa, 2011).

Renal transplantation is required to take life-long immunosuppressive medication to impede graft rejection. Non adherence is behavioral rather than medical issue. Non adherence to immunosuppressive medication and life style modification is common issue and increases over time. Both dosage and timing of medication are vital. Failure to take medication as prescribed is a hazard factor for acute or late rejection and graft loss and patient mortality (Vlaminck, 2004). Non-adherence is a serious health concern among renal transplant recipients. Although IST (Immunosuppressant therapy) adherence is critical to graft maintenance and survival, a recent meta-analysis revealed that IST non-adherence rate of 36\% among renal transplant recipients (Dew et al., 2007). The potential consequences of non-adherence include graft failure, return to dialysis, retransplantation, and death. Approximately $35 \%$ of renal graft loss is due to IST non-adherence, and the odds of graft failure increase seven-fold in non-adherent compared with adherent renal transplant recipients. Moreover, life expectancy for non-adherent renal transplant recipients is four years less than that of adherent renal transplant recipient (Butler et al., 2004 \& Chisholm-Burns, Spivey \& Wilks, 2009).

Specific lifestyle behavior can help to reduce such risks caused by immunosuppressive medication as increase risk of infection so renal recipient need good hygienic care to prevent and control infection. In addition to unwanted weight gain, hyperglycemia, hypertension, osteoporosis, and muscle atrophy they advised to do regular physical exercises and be aware of beneficial eating habits and calories intake (Gheith et al., 2008). To confirm the success of transplantation in the long term, it is important that the nursing staff work to educate these patients, ensuring that they return to their homes with enough knowledge, to keep the graft and appropriate self-help skills and knowledge, this include the proper use of medication and it s side effects, addressing questions about adherence to the therapeutic regimen, since not following the therapy increases the risk of graft loss; understanding of the care for prevention and identification of signs and symptoms of infection and rejection, measuring vital signs, weight and glucose test, the importance of balanced and healthy diet associated with exercise to prevent weight gain, skin care since the immunosuppressive regimen increase the risk of development of malignancies as well as issues of fertility and lifestyle (Trevit, et al., 2012 \& Amaral et al., 2013). So this study will be carried out to assess the lifestyle modification among renal transplant recipients. Aim of the study The aim of this study was assess the lifestyle modification among renal transplanted recipients.

\section{Study design}

\section{Material And Methods}

Cross sectional study was used to carry out this research.

\section{Setting}

The study was conducted at outpatient clinic at Nephrology and Urology Center affiliated to Mansoura University.

\section{Subjects}

The study included patients carried out renal transplantation one year and more, aged from 18 -65 years as renal transplantation did not carried out for patients exceed age of 60 (Adult stage from 18 to 65 years) ${ }^{(2)}$.

\section{Sampling technique and sampling size}

A simple random sample was used as a sampling technique selected from the record belonged to post renal transplant recipients at Nephrology and Urology Center affiliated from Mansura University.

To calculate sample size, the DSS research.com sample size calculator will be used, comparing the previous study results of good adherence to immunosuppressive medication that was $97 \%{ }^{(3)}$ ) and the wanted level will be $100 \%$ at $\alpha$ error $5 \%$ (95\% significance) and $\beta$ error $20 \%, 80 \%$ power of the study, so this study required a sample size of 120 patients

\section{Study tools}

After reviewing the relevant literatures five tools were used in this study for data collection.

\section{Tool I: Structure interview to assess socio demographic and economical characteristics of renal transplant recipients (Appendix II)}

This tool was consisted of eight items to assess socio demographic and economical characteristics of the renal transplant recipients including: six, age, residence, educational level, occupation, marital status, number of children pre and post renal transplantation, and monthly income. 


\section{Tool II: Health profile of renal transplant recipients (Appendix III) It Included Four Parts As The Following:}

1 - Past and current health history which including 11 items,

contained: Cause of renal failure, duration and type of dialysis, history of other chronic disease, relative suffering from renal failure, duration since renal transplantation, kidney source, signs and symptoms of rejection, complication after transplantation, and drug used after transplantation.

2- Physical examination that contained eight items: vital signs (body temperature, plus, respiration, and blood pressure), height, weight, body mass index and urine output.

3- Signs and symptoms related to side effects of immunosuppressive medications it consisted of 14 items: mouth ulcer, skin papules, and pigmentation, burning sensation in hands and foots, face edema, hair in face and body (hirsutism), loss head hair (alopecia), burning sensation during urination/presence of lower abdominal pain during urination, vision diminution, cataract, blurring vision, and stomach acidity.

4- Laboratory investigations that contain five items: Kidney function test, liver function test, immunosuppressive medications level, urine analysis and CBC.

\section{Tool III: Structured Interview To Assess Renal Transplant Recipients' Lifestyle Modification (Appendix} IV)

It would assess renal transplant recipients' lifestyle modification related to eight domains as the following:

1- Dietary habits: six items asked about; number of meals daily, food between meals, type of cooking, type of oil, food avoided post transplantation, and recommended foods post transplantation (6 items).

2- Drinking habits: two item asked about; amount and types of drinks.

3- Smoking habits: two item asked about; kinds and duration of smoking.

4- Follow up: two items asked about; time of follow up and cause of didn't attendance.

5- Sexual and reproductive health for both male and female;

a. For both unmarried males and females, two items about consulting before marriage and marriage duration.

b. For married males, two items about presence of sexual problems, and their behaviors toward these problems.

c. For both married and unmarried females, one item about return of menstrual cycle

d. For married females, four items about problems related to sexual activities, consultation before pregnancy, usage of contraceptive method, and duration of usage.

6- Physical activity: two items asked about; kind and duration of exercise they were practiced.

7- Rest and sleep: four items asked about; sleeping hours daily, improvement of sleeping post renal transplantation, problems during sleep and sleeping on abdomen and graft site.

8- Stress management: one item asked about how renal transplant recipients manage stress.

\section{Tool IV: Structure interview to assess renal transplant recipients' immunosuppressive medication} adherence (Appendix V)

It consisted of 17 statements were represented as a four points Likart scale ranging from zero (never), one (sometimes), two (often), to three (always), if the statements were negative the scoring system was reversed in SPSS three (never), two (sometimes), one (often), to three (always), to determine the level of adherence of the renal transplant recipients to the recommended behaviors toward administration of immunosuppressive medications the score ranging between 0\% (never taken as prescribed) to 100\% (always taken as prescribed).

Statements defined as: Negative adherence including: missed one or two doses of immunosuppressive medication during holidays, and vacations, underestimate the number of times taking immunosuppressive medication, stop immunosuppressive medication without consulting the doctor, and underestimate prescribed dose. Positive adherence including: remember forgotten doses of the medication, taking immunosuppressive medications regularly as doctor ordered, know medications in terms of its name, know medications in terms of form, know medications in terms of their color, know medications in terms of dosing prescribed, take forgotten dose as soon as remembered and take other dose on it's time, taking immunosuppressive medications before eating, check the level of immune therapy after 12 hours of the past dose, arrange the time of taking medications, with eating times or any favorite times, consult physician when changing the time of immunosuppressive medications, consult the doctor when changing the doses of medications, and put the immunosuppressive medications in appropriate place.

\section{Scoring system}

The total scores of the adherence ranged from 0 to 51 . The adherence level was categorized into 4 categories:

- Never or non adherent less than $51 \%$ - of total score $(<25.5)$.

- Poor adherent $=51 \%$ to less than $71 \%$ of total sore $(25.5-<36.21)$.

- Partial adherent; $71 \%$ to less than $96 \%$ of total score $(>36.21-<48.96)$.

- Good adherent more than $96 \%$ - 100\% of total score ( $\geq 48.96-51)$. 


\section{Tool V: structure interview to assess renal transplant recipients' compliance (Appendix VI)}

It was represented as a four points Likart scale ranging from zero (never or non-compliant), one (poor compliant), two (partial compliant), to three (good compliant), to determine the level of compliance of the renal transplant recipients to the recommended lifestyle behaviors. Scores will be recorded between zero and 100 , score less than 51 will be defined as never or noncompliant; 51 to less than 71 poor compliant; 71 to less than 96 partial compliant; and 96 to 100 good compliant (Gheith et al., 2008).

It consisted of 11 categories as the following:

- Medication consists of 10 items $=30$ marks.

- Dietary recommendation consist of 10 items $=30$ marks.

- Follow up of fluid, body temperature and daily weight consist of 9 items $=27$ marks.

- Avoiding infection consist of 13 items $=39$ marks.

- Follow up visits consist of 9 items $=27$ marks.

- Social relationships consist of 8 items $=24$ marks.

- Work post renal transplantation consist of 3 items $=9$ marks

- Daily physical exercise consist of 4 items $=12$ marks.

- Marital relation consists of three points as the following:

1. For males and female consist of one item $=3$ marks.

2. For married males consist of 3 items $=9$ marks .

3. For married females consist of 9 items $=27$ marks.

- Precaution of skin cancer consists of 4 items $=12$ marks.

- Precaution of female breast cancer consists of 3 items $=12$ marks

- Smoking consist of 4 items $=6$ marks for patient who don't smoke and 12 marks for previous smoking.

\section{Scoring system}

The total scores of post renal transplant recipients ranged from 0 to 270 .

Score for single male $=225$

- Never or non-compliant; less than $51 \%$ - of total scores $(\leq 112.5)$

- Poor compliant $=\geq 51 \%$ to less than $71 \%$ of total sores $(\geq 112.5-157.5)$

- Partial compliant $=\geq 71 \%$ to less than $96 \%$ of total scores $(\geq 157.5-216)$

- Good compliant $\geq 96 \%-100 \%$ of total score ( $\geq 216-225)$

Score for single female $=234$

- Never or non-compliant; less than $51 \%$ - of total scores $(<117)$

- Poor compliant $=\geq 51 \%$ to less than $71 \%$ of total scores $(\geq 117-163.5>)$

- partial compliant; $\geq 71 \%$ to less than $96 \%$ of total scores $(\geq 163.5-<224)$

- Good compliant $\geq 96 \%-100 \%$ of total score ( $\geq 224-234$ )

Score for married male $=234$

- Never or non-compliant; less than $51 \%$ - of total scores $(<117)$.

- Poor compliant $=\geq 51 \%$ to less than $71 \%$ of total sores $(\geq 117-<163.5)$.

- Partial compliant; $\geq 71 \%$ to less than $96 \%$ of total score $(\geq 163.5-<224)$.

- Good compliant $\geq 96 \%-100 \%$ of total score ( $\geq 224-234)$.

Score for married female $=261$

- Never or non-compliant $;<51 \%$ - of total scores $(<130)$.

- Poor compliant $=\geq 51 \%$ to $<71 \%$ of total scores $(130 \geq-<182.7)$.

- Partial compliant; $\geq 71 \%$ to $<96 \%$ of total scores $(\geq 182.7-<250)$.

- Good compliant $\geq 96 \%-100 \%$ of total score $(\leq 250-261)$.

\section{Methods}

This study was accomplished throughout two main stages

I- Preparatory stage:

1-Administrative process

An official letter was issued from the Faculty of Nursing Mansoura University to the director of Nephrology and Urology Center affiliated to Mansura University to permit the researcher to carry out the study. 


\section{2-Developing of the study tools}

Tools of data collection were developed by the researcher based on reviewing the relevant literature except fifth tool was adopted from (Gheith et al., 2008).

1-A pilot study was conducted on $10 \%$ of studied sample (12 patients) selected randomly from the same setting and excluded from the studied sample to evaluate the clarity, applicability, and reliability of the research tools and estimate the approximate time required for data collection. Accordingly the necessary modification was done, some questions were added and others were clarified or omitted.

2-Validity testing was done to the tools by submitting the tools to five expertises in the field of "community health nursing, nursing in addition to statistics ". Their recommended modifications had been done.

3-Reliability of tools was tested by using Cronbach's alpha test in SPSS v.16 as the following:

- The Cronbach's alpha was 0.858 for renal transplant recipients' socio demographic and economical characteristics.

- The Cronbach's alpha was 0.870 for renal transplant recipients' health profile.

- The Cronbach's alpha was 0.780 for renal transplant recipients' lifestyle modification.

- The Cronbach's alpha was 0.758 for renal transplant recipients' adherence to immune suppressive medication.

- The Cronbach's alpha was 0.871 for renal transplant recipients' compliance.

\section{1-Data collection}

\section{II- Operational stage}

- The duration of data collection approximately 12 weeks from 1/9/2015 to 30/11/2015.

- The researcher attending the outpatient clinic at Saturday, Wednesday per week from 9:00 am to 12:00 afternoon started by introducing herself to the renal transplant recipients and giving them a brief orientation about the aim of the study.

2-The research was interviewed with the renal transplant recipients to collect the data by using the prepared study tools and each tool take from 5 to 7 minutes to be fulfilled.

3-Ethical consideration

- An approval was obtained from Research Ethical Committee, Faculty of Nursing Mansoura University to accomplish this study.

- An approval was obtained from the renal transplant recipients. The researcher introduced herself and a simple explanation about the aim of the study would be given to them. They assured that their participation in the study was voluntary and that collected data would treat confidentially and would be only used for the purpose of the study. Renal transplant recipient would be informed that they had the right to withdraw at any time from the study.

\section{4-Statistical analysis}

- Data were sorted, coded, organized, categorized and then transferred into especially designed formats.

- Data were analyzed using SPSS (Statistical Package for Social Sciences) version 16 stands for statistical product and service solution.

- Data were presented by using descriptive statistics in the form of frequencies and percentage.

- $\quad$ The qualitative (categorical variables) were compared using chi square $\left(\mathrm{x}^{2}\right)$ test and montcarlo (MC) exact $\left(\mathrm{x}^{2}\right)$ test was used when these is cells with expected value less than 5.

- While quantitative variables were compared using (t) test if two groups and one way ANOVA (F) test if more than two groups. The differences was considered significant at $\mathrm{p} \leq 0.05$

\section{Results}

Table (1) presents socio-demographic and economic characteristics of renal transplant recipients, their age ranged from 18 to up to 58 years, with a mean age of $34.11 \pm 7.08$ years. More than two third $(68.3 \%)$ and more than half $(59.2 \%)$ of them were males and married respectively. As regarded to educational level and occupation more than half $(55.0 \%)$ and more than one third $(36.7 \%)$ of renal transplant recipients were secondary education and didn't work respectively. The majority $(83.3 \%)$ of them were resident at rural area. In relation to monthly income more than half $(53.3 \%)$ of renal transplant recipients earned $1500 \rightarrow 2000$ pounds with mean of $1652.08 \pm 318.56$ pounds.

Table (2) shows that, more than half (54.2\%) of renal transplant recipients were suffering from renal failure for 6 years. Concerning causes of renal failure the majority $(85.8 \%)$ of them had unknown cause of renal failure, more than three fourths $(78.3 \%$ )of renal transplant recipients were on hemodialysis, less than half $(48.3 \%)$ of them stayed on dialysis for at least one year. Other chronic diseases were reported in $15.0 \%, 5.0 \%$ and $1.7 \%$ of renal transplant recipients including: hypertension, DM, HCV and HBV respectively. Less than one 
third (30.8\%) of them had renal transplantation from 2-6years ago. The majority (85.8\%) of renal transplant recipients had related kidney donor. Concerning the signs and symptoms of rejection, only $5.8 \%$ of them suffered from increased serum creatnine as a symptom of rejection. Regarding the post renal transplantation drugs used all of renal transplant recipients taken immunosuppressive medications. Table (3) illustrates physical examination and laboratory investigations of renal transplant recipients. Less than half $(46.7 \%)$ had hypertension and only $2.5 \%$ of them had hypotension post transplantation. shows smoking habits; follow up, Regarding urine output two thirds $(66.6 \%)$ of renal transplant recipients had $1.5-3 \mathrm{~L}$ urine per day with mean $3.00 \pm 0.63 \mathrm{cc}$. concerning body mass index the majority $(85.8 \%)$ of them were overweight (more than $30 \mathrm{Kg} / \mathrm{m}^{2}$ ). Regarding laboratory investigations the entire renal transplant recipients had normal kidney function test, liver function test, $\mathrm{CBC}$, immunosuppressive medications level test and urine analysis test.

Table (4) Illustrates side effects related to immunosuppressive medications among renal transplant recipients. Less than two thirds (62.5\%) of them had minor and major side effects related to immunosuppressive medications. One eighth (12.5\%) of renal transplant recipients were suffered from skin papules as a minor side effect. While11.7 of them suffered from vision diminution as major side effects. Table (5) reveals dietary and drinking habits of renal transplant recipients. Regarding dietary habit the majority $(83.3 \%)$ of them had three meals per day, less than one third (30.8\%)of renal transplant recipients had fast food and sandwich between meals. Concerning type of cooking the majority (89.2) of them had fried food. Regarding food avoided post renal transplantation $85.8 \%, 70.8 \%, 88.3 \%$ and $80.8 \%$ of renal transplant recipients avoided salted, canned, sugary food and uncooked respectively. Regarding type of fats used most (92.0\%) of them used animal oil. Concerning recommended food $92.5 \%, 95.8 \%, 92.5 \%$ and $99.2 \%$ recommended to had white meats as: chicken, fruits, beans and rice respectively. Finally more than three fourths (78.3\%) of them drank more than 2.5 liter of water per day

Table (6) shows smoking habits; follow up, physical activity, sleeping and stress managements as a lifestyle of the post renal transplant recipients.Only $12.5 \%$ of renal transplant recipients were cigarette smoker. Concerning follow up less than two thirds(63.3\%) of them reported that they attended for follow up every 3 months the entire of renal transplant recipients didn't come when there was enough medication. Regarding physical activities two fifths (40.0\%) of renal transplant recipients practiced physical activities, $35.0 \%$ of them walk and three fourths (75. \%) ofrenal transplant recipients practice exercise for half an hour daily. Regarding sleep more than three fourths $(78.3 \%)$ of them stated that sleep was better post transplantation, two thirds, $(68.4 \%)$ of renal transplant recipients were rested and slept 8 hours per day and $81.7 \%$ of them slept on the abdomen or on the side of graft. Concerning stress management almost two thirds $(65.0 \%)$ of renal transplant recipients visited their relatives when faced any stress situations.

Table (7) reveals sexual and reproductive health of renal transplant recipients. More than three fourths (77.5\%) of renal transplant recipients consulting before marriage and only8.16\% of males had week erection, all $(100.0 \%)$ of them consulted the doctors when there is sexual problems. Concerning females reproductive health less than two thirds $(60.5 \%)$ of them regained menstruation within one month post transplantation. All (100\%) females consulted doctors before pregnancy and more than half (51.8\%) of renal transplant recipients used contraceptive method (Pills and local).Regarding number of children of renal transplant recipients among males and females pre-and post renal transplantation, number of children among males were equal pre-and post transplantation $53.1 \%$, while in female number of children pre-transplantation were $72.7 \%$ while $40.9 \%$ post transplantation with no statistically significant differences, $\mathrm{p}<0.05$.

Table (8) illustrates renal transplant recipients' adherence to immunosuppressive medication with median score 51.0\%. Most (99.2\%) of renal transplant recipients never stop immunosuppressive medications without consulting the doctor and underestimate prescribed dose. In addition most (99.2\%) of them always consult the physician when changing the time, doses, and put it in appropriate place. While minority (7.5\%) of renal transplant recipients had never toke immunosuppressive medication before eating. Figure (1) explains the total level of adherence to immunosuppressive medication among renal transplant recipients, the majority $(80.0 \%)$ of them had a good adherence to immunosuppressive medication post renal transplantation.

Table (9) presents level of renal transplant recipients compliance regarding medications and dietary recommendation; more than one third $(39.2 \%)$ and less than one forth $(23.3 \%)$ of them respectively had a good compliance with median score of 28.0, and 27.0 respectively. In relation to follow up of fluid, body temperature, and daily weight more than two thirds $(68.3 \%)$ of renal transplant recipients were partial compliant with median score 20.0. As regards to avoiding infection the majority (82.5\%) of renal transplant recipients were partially compliant with median score of 34.0.Regarding follow up visits less than three fourths (70 \%) of renal transplant recipients were partially compliant with median score of 24.0. Towards to social relation; more than three fourths $(75.8 \%)$ of renal transplant recipients had a good compliance with median score of 24.0. Regarding work post renal transplantation half $(50.0 \%)$ of renal transplant recipients had a good of compliant with median score of 8.5. In relation to physical exercise less than two thirds (60\%) of renal transplant recipients were noncompliant with median score of 6.0.Concerningmarital for male and female the majority $(85.2 \%)$ of renal 
transplant recipients had a good a compliance with median score of 3.0.Towards marital relation for males more than one third $(34.3 \%)$ of renal transplant recipients had a good compliance with median score of 6.0.

While in female marital relation, one third $(33.3 \%)$ of renal transplant recipients were good compliant with median score of 24.0. In relation to precaution of skin cancer, more than three fourths (75.8\%) of the renal transplant recipients were non- compliant with median score of 5.0. Furthermore, precaution of females breast cancer; the majority $(89.5 \%)$ of female renal transplant recipients had non-compliant with median score of 3.0.Finallyrelated to smoking; less than half $(45.8 \%)$ of renal transplant recipients had non-compliant with median score of 12.0 .

Figure (2) explains that the majority (87.5\%) of renal transplant recipients were partially compliant.

Table (10) presents that there was statistically significant association between sex, marital status, and occupation of renal transplant recipients, and their total adherence score to immunosuppressive medication $\mathrm{p}=$ $0.023,0.005$ and 0.046 respectively.

Table (11) summarizes the association between total score of renal transplant recipients'compliance, and socio demographic characteristics. There were statistically significant association between age, marital status, occupation, and residence of renal transplant recipients, and their total compliance score, $p=0.026,0.00$, 0.288 and 0.015 respectively. Figure (3) illustrates significant correlation between renal transplant recipients' total adherence score, and their total compliance score $(r=+0.272 \& \mathrm{p}=0.003)$.

Table (1) Renal transplant recipients' socio- demographic and economic characteristics (n-120).

\begin{tabular}{|c|c|c|}
\hline Item & No & $\%$ \\
\hline \multicolumn{3}{|l|}{ Age (years) } \\
\hline $18->28$ & 66 & 55.0 \\
\hline $28->38$ & 44 & 36.7 \\
\hline $38->48$ & 8 & 6.7 \\
\hline $48-\geq 58$ & 2 & 1.7 \\
\hline Mean \pm SD & $34.11 \pm 7.08$ & \\
\hline \multicolumn{3}{|l|}{ sex } \\
\hline Male & 82 & 68.3 \\
\hline Female & 38 & 31.7 \\
\hline \multicolumn{3}{|l|}{ Marital status } \\
\hline Married & 71 & 59.2 \\
\hline Single & 46 & 38.3 \\
\hline Divorce & 3 & 2.5 \\
\hline \multicolumn{3}{|l|}{ Level of education } \\
\hline $\begin{array}{l}\text { Illiterate } \\
\text { Int }\end{array}$ & 9 & 7.5 \\
\hline Read and write & 18 & 15.0 \\
\hline Secondary & 66 & 55.0 \\
\hline University & 27 & 22.5 \\
\hline \multicolumn{3}{|l|}{ Occupation } \\
\hline Didn't work & 44 & 36.7 \\
\hline Administrative work & 31 & 25.8 \\
\hline Professional & 23 & 19.2 \\
\hline Trade work & 16 & 13.3 \\
\hline Manual work & 6 & 5.0 \\
\hline \multicolumn{3}{|l|}{ Residence } \\
\hline Rural & 100 & 83.3 \\
\hline Urban & 20 & 16.7 \\
\hline \multicolumn{3}{|l|}{ Monthly income } \\
\hline $1000->1500$ & 43 & 35.8 \\
\hline $1500->2000$ & 64 & 53.3 \\
\hline$\geq 2000$ & 13 & 10.8 \\
\hline Mean \pm SD & \multicolumn{2}{|c|}{$1652.08 \pm 318.56$} \\
\hline
\end{tabular}

Table (2): Past and current health history of renal transplant recipients $(n=120)$

\begin{tabular}{|l|c|c|}
\hline Items & No & $\%$ \\
\hline Duration of renal failure & 56 & 54.2 \\
$>6$ year & 37 & 30.8 \\
$6->10$ years & 18 & 15.0 \\
\hline$<10$ years & & \\
\hline Cause of renal failure & 103 & 85.8 \\
Unknown & 10 & 8.3 \\
Hypertension & 7 & 5.8 \\
Polycystic, Tuberculosis and kidney reflux & & \\
\hline Type of dialysis & 94 & 78.3 \\
Heamodialysis & 5 & 4.1 \\
Peritoneal & & \\
\hline
\end{tabular}




\begin{tabular}{|c|c|c|}
\hline $\begin{array}{l}\text { Duration of dialysis (years) } \\
<1 \\
1-<6 \\
\geq 6\end{array}$ & $\begin{array}{c}58 \\
32 \\
9\end{array}$ & $\begin{array}{c}48.3 \\
26.7 \\
7.5\end{array}$ \\
\hline $\begin{array}{l}\text { History of other chronic disease } \\
\text { Hypertension } \\
\text { Diabetes mellitus } \\
\text { Hepatitis C virus, Hepatitis B virus }\end{array}$ & $\begin{array}{c}18 \\
6 \\
2 \\
\end{array}$ & $\begin{array}{l}15.0 \\
5.0 \\
1.7\end{array}$ \\
\hline $\begin{array}{l}\text { Relative suffering from renal failure } \\
\text { Mother, Father and Grand father }\end{array}$ & 4 & 4.1 \\
\hline $\begin{array}{l}\text { Duration since renal transplantation } \\
\leq 2 \text { year } \\
>2-\geq 6 \text { years } \\
>6-\geq 10 \text { years } \\
>10 \text { years }\end{array}$ & $\begin{array}{l}32 \\
37 \\
33 \\
18\end{array}$ & $\begin{array}{l}26.7 \\
30.8 \\
27.5 \\
15.0\end{array}$ \\
\hline $\begin{array}{l}\text { Source of kidney donor } \\
\text { Related donors } \\
\text { Unrelated donors }\end{array}$ & $\begin{array}{c}103 \\
17\end{array}$ & $\begin{array}{c}85.8 \\
14.16\end{array}$ \\
\hline Signs and symptoms of rejection & 7 & 5.8 \\
\hline $\begin{array}{l}\text { Complications post transplantation } \\
\text { Hypertension } \\
\text { Diabetes mellitus } \\
\text { Pneumonia } \\
\text { Cardiac embolism }\end{array}$ & $\begin{array}{c}34 \\
15 \\
6 \\
3\end{array}$ & $\begin{array}{c}28.3 \\
12.3 \\
5.0 \\
2.5\end{array}$ \\
\hline $\begin{array}{l}\text { Drugs used post transplantation* } \\
\text { Immunosuppressive } \\
\text { Vitamins } \\
\text { Anti acids } \\
\text { Anti diabetic } \\
\text { Anti hypertension } \\
\end{array}$ & $\begin{array}{l}120 \\
116 \\
111 \\
35 \\
15\end{array}$ & $\begin{array}{l}100.0 \\
96.7 \\
92.5 \\
29.2 \\
12.5 \\
\end{array}$ \\
\hline
\end{tabular}

*More than one answer

Table (3) Physical examination and laboratory investigations of the renal transplant recipients ( $\mathrm{n}=120)$

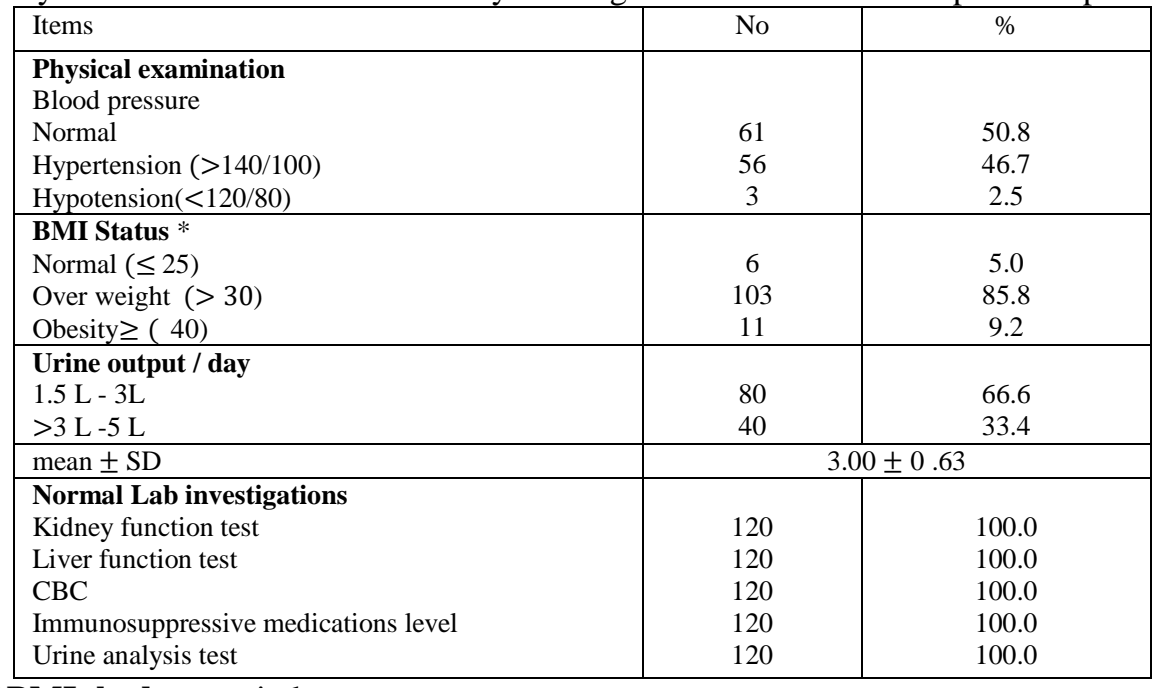

* BMI: body mass index

Table (4) Side effects related to immunosuppressive medications among renal transplant recipients $(n=120)$

\begin{tabular}{|c|c|c|}
\hline Items & No & $\%$ \\
\hline Minor & & \\
\hline Skin papules & 15 & 12.5 \\
\hline Face edema & 12 & 10.0 \\
\hline Loss head hair (alopecia) & 7 & 5.8 \\
\hline Burning sensation of hand, foot & 6 & 5.0 \\
\hline Increase hair in face and body & 4 & 3.3 \\
\hline Stomach acidity & 4 & 3.3 \\
\hline Burning urination/Lower abdominal pain during urination & 2 & 1.6 \\
\hline Major & & \\
\hline Vision diminution & 14 & 11.7 \\
\hline Cataract & 5 & 4.2 \\
\hline Blurring vision & 6 & 5.0 \\
\hline
\end{tabular}




\begin{tabular}{|l|c|}
\hline Total & 62.5 \\
\hline
\end{tabular}

Table (5) Dietary and drinking habits as a lifestyle of the renal transplant recipients $(\mathrm{n}=120)$

\begin{tabular}{|c|c|c|}
\hline \multirow{2}{*}{\multicolumn{3}{|c|}{$\begin{array}{l}\text { Items } \\
\text { Number of meals/day }\end{array}$}} \\
\hline & & \\
\hline Three & 100 & 83.3 \\
\hline$>$ Three & 20 & 16.7 \\
\hline \multicolumn{3}{|l|}{ Food between meals } \\
\hline Fast food/Sandwich & 37 & 30.8 \\
\hline Juice & 17 & 14.2 \\
\hline Fruits/vegetables & 9 & 7.5 \\
\hline Cooked & 5 & 4.2 \\
\hline \multicolumn{3}{|l|}{ Type of food cooking* } \\
\hline Grilled & 113 & 94.2 \\
\hline Fried & 111 & 92.5 \\
\hline Boiled & 107 & 89.2 \\
\hline \multicolumn{3}{|c|}{ Prevented food post transplantation* } \\
\hline Sugary food & 106 & 88.3 \\
\hline Salted & 103 & 85.8 \\
\hline Un cooked & 97 & 80.8 \\
\hline Canned & 85 & 70.8 \\
\hline \multicolumn{3}{|l|}{ Type of fats } \\
\hline Animal oil & 111 & 92.5 \\
\hline Plant oil & 5 & 4.2 \\
\hline \multicolumn{3}{|c|}{ Recommended foods post transplantation* } \\
\hline Rice & 119 & 99.2 \\
\hline Fruits & 115 & 95.8 \\
\hline Beans & 111 & 92.5 \\
\hline White meats as Chicken & 111 & 92.5 \\
\hline \multicolumn{3}{|l|}{ Drinks/day } \\
\hline \multicolumn{3}{|l|}{ Water } \\
\hline $1.5-2 \mathrm{~L}$ & 4 & 3.3 \\
\hline$<2-\geq 2.5 \mathrm{~L}$ & 22 & 18.3 \\
\hline$>2.5 \mathrm{~L}$ & 99 & 78.3 \\
\hline \multicolumn{3}{|l|}{ Tea (cups) } \\
\hline 1 & 18 & 15.0 \\
\hline 2 & 26 & 21.7 \\
\hline 3 & 28 & 23.3 \\
\hline \multicolumn{3}{|l|}{ Coffee (cups) } \\
\hline $1-2$ cup & 23 & 19.1 \\
\hline \multicolumn{3}{|l|}{ Cola (cups) } \\
\hline 1 & 7 & 5.8 \\
\hline 2 to 3 & 6 & 5 \\
\hline
\end{tabular}

* More than one answer

Table (6) Smoking, follow up, physical activity, rest, sleep and stress management as a lifestyle of the renal transplant recipients $(n=120)$

\begin{tabular}{|l|c|c|}
\hline Items & No & $\%$ \\
\hline Cigarettes smoking & 15 & 12.5 \\
\hline Follow up schedule & 2 & 1.7 \\
Weekly & 37 & 30.8 \\
Monthly & 104 & 67.5 \\
More than 3 month & & \\
\hline Reason for didn't attending for follow up & 120 & 100.0 \\
Having enough medication & & \\
\hline Types of physical activities & 42 & 35.0 \\
Walking & 6 & 5.0 \\
Light exercises & & \\
\hline Exercise duration/day & 36 & 75.0 \\
Half an hour & 12 & 25.0 \\
One hour & 1 & 0.8 \\
\hline Rest and sleep hours/day & 82 & 68.4 \\
<8 hours & 37 & 30.8 \\
\hline hours & 94 & 78.3 \\
\hline S hours & & \\
\hline Sleep pattern is better after transplantation & 20 & 16.7 \\
\hline Problems during sleep & & \\
Acidity & & \\
\hline
\end{tabular}




\begin{tabular}{|l|c|c|}
\hline Mouth dryness & 8 & 6.7 \\
\hline Sleep site & & \\
On the site of graft & 98 & 81.7 \\
\hline Stress managements & 78 & 65.0 \\
Visiting relatives & 36 & 30.0 \\
Sleep & 3 & 2.5 \\
Practice sporting & 3 & 2.5 \\
Reading stores and Quran & \\
\hline
\end{tabular}

Table (7) Sexual and reproductive health of the renal transplant recipients $(\mathrm{n}=120)$

\begin{tabular}{|c|c|c|c|c|}
\hline \multicolumn{3}{|l|}{ Items } & No & $\%$ \\
\hline \multicolumn{3}{|l|}{ Consulting before marriage } & 93 & 77.5 \\
\hline \multicolumn{3}{|l|}{ Time of marriage } & & \\
\hline \multicolumn{3}{|l|}{ Before transplantation } & 48 & 40.0 \\
\hline \multicolumn{3}{|l|}{ After transplantation $\geq 1$ year } & 26 & 21.6 \\
\hline \multicolumn{5}{|l|}{$\begin{array}{l}\text { For males } n=49 \\
\text { sexual problem }\end{array}$} \\
\hline \multirow{2}{*}{\multicolumn{3}{|c|}{$\begin{array}{l}\text { Week erection } \\
\text { Behave with sexual problem }\end{array}$}} & 4 & 8.16 \\
\hline & & & & \\
\hline \multicolumn{3}{|l|}{ Consult doctors } & 49 & 100.0 \\
\hline \multirow{2}{*}{\multicolumn{3}{|c|}{ For female $(n=38)$}} & & \\
\hline & & & & \\
\hline \multicolumn{3}{|l|}{$\begin{array}{l}\text { Return of menstrual cycle } \\
<\text { One month }\end{array}$} & 7 & 18.4 \\
\hline \multicolumn{3}{|l|}{ One month } & 23 & 60.5 \\
\hline \multicolumn{3}{|l|}{$>$ One month } & 8 & 21.1 \\
\hline \multicolumn{3}{|c|}{ Problems related to sexual activities $(n=22)$} & & \\
\hline \multicolumn{3}{|c|}{ Urinary tract infection } & 9 & 40.9 \\
\hline \multicolumn{3}{|l|}{ Consultation before pregnancy } & 22 & 100.0 \\
\hline \multicolumn{3}{|l|}{ Contraceptive method } & & \\
\hline \multicolumn{3}{|l|}{ Pills and local } & 14 & 63.6 \\
\hline \multicolumn{3}{|l|}{ Duration of usage } & & \\
\hline \multicolumn{3}{|l|}{$<1$ year } & 4 & 18.1 \\
\hline \multicolumn{3}{|l|}{$1-3$ years } & 6 & 27.2 \\
\hline \multicolumn{3}{|l|}{$>3$ years } & 4 & 18.1 \\
\hline \multirow[t]{2}{*}{ Number of children } & \multicolumn{2}{|l|}{ Male $n=49$} & Fen & \\
\hline & No & $\%$ & No & $\%$ \\
\hline \multirow{2}{*}{$\begin{array}{l}\text { Before transplantation } \\
\text { After transplantation }\end{array}$} & 26 & 53.1 & 16 & 72.7 \\
\hline & 27 & 55.1 & 9 & 40.9 \\
\hline Significant test & $\begin{array}{l}x^{2}=1.53 \\
p=0.216\end{array}$ & & & \\
\hline
\end{tabular}

Table (8) Adherence to immunosuppressive medication among the renal transplant recipients $(\mathrm{n}=120)$

\begin{tabular}{|c|c|c|c|c|c|c|c|c|}
\hline \multirow[t]{2}{*}{ Items } & \multicolumn{2}{|c|}{ Never } & \multicolumn{2}{|c|}{ Some times } & \multicolumn{2}{|c|}{ Often } & \multicolumn{2}{|c|}{ Always } \\
\hline & No & $\%$ & No & $\%$ & No & $\%$ & No & $\%$ \\
\hline \multicolumn{9}{|c|}{ Negative adherence } \\
\hline $\begin{array}{l}\text { Missed one or two doses of } \\
\text { immunosuppressive medication } \\
\text { during holidays and vacations }\end{array}$ & 75 & 62.5 & 28 & 23.3 & 16 & 13.3 & 1 & 0.8 \\
\hline $\begin{array}{l}\text { Underestimate the number of } \\
\text { times taking immunosuppressive } \\
\text { medication }\end{array}$ & 118 & 98.3 & 1 & 0.8 & 1 & 0.8 & 0 & 0 \\
\hline $\begin{array}{l}\text { Stop immunosuppressive } \\
\text { medication without consulting } \\
\text { the doctor }\end{array}$ & 119 & 99.2 & 1 & 0.8 & 0 & 0 & 0 & 0 \\
\hline Underestimate prescribed dose & 119 & 99.2 & 1 & 0.8 & 0 & 0 & 0 & 0 \\
\hline \multicolumn{9}{|c|}{ Positive adherence } \\
\hline $\begin{array}{l}\text { Remember forgotten doses of the } \\
\text { medication }\end{array}$ & 0 & 0 & 5 & 4.5 & 4 & 3.3 & 111 & 92.5 \\
\hline $\begin{array}{l}\text { Taking immunosuppressive } \\
\text { medication regularly as doctor } \\
\text { ordered }\end{array}$ & 0 & 0 & & & 2 & 1.7 & 118 & 98.3 \\
\hline $\begin{array}{l}\text { Know medication in terms of its } \\
\text { name }\end{array}$ & 0 & 0 & 1 & 0.8 & 1 & 0.8 & 118 & 98.3 \\
\hline $\begin{array}{l}\text { Know medication in terms of } \\
\text { form }\end{array}$ & & 0 & 1 & 0.8 & 1 & 0.8 & 118 & 98.3 \\
\hline $\begin{array}{l}\text { Know medication in terms of } \\
\text { their color }\end{array}$ & 0 & 0 & 1 & 0.8 & 1 & 0.8 & 118 & 98.3 \\
\hline
\end{tabular}


Lifestyle Modification Among Post Renal Transplant Recipients

\begin{tabular}{|l|c|c|c|c|c|c|c|c|}
\hline $\begin{array}{l}\text { Know medication in terms of } \\
\text { dosing prescribed }\end{array}$ & 0 & 0 & 1 & 0.8 & 1 & 0.8 & 118 & 98.3 \\
\hline $\begin{array}{l}\text { Take forgotten dose as soon as } \\
\text { remembered and take other } \\
\text { dose on its time }\end{array}$ & 0 & 0 & 1 & 0.8 & 3 & 2.5 & 116 & 96.7 \\
\hline $\begin{array}{l}\text { Taking immunosuppressive } \\
\text { medication before eating }\end{array}$ & 9 & 7.5 & 8 & 6.7 & 18 & 15.0 & 85 & 70.8 \\
\hline $\begin{array}{l}\text { Check the level of immune } \\
\text { therapy after 12 hours of the past } \\
\text { dose }\end{array}$ & 1 & 0.8 & 1 & 0.8 & 3 & 2.5 & 115 & 95.8 \\
\hline $\begin{array}{l}\text { Arrange the time of taking } \\
\text { medication, with eating times or } \\
\text { any favorite times }\end{array}$ & 1 & 0.8 & 2 & 1.7 & 2 & 1.7 & 115 & 95.8 \\
\hline $\begin{array}{l}\text { Consult my physician when } \\
\text { changing the time of } \\
\text { immunosuppressive medication }\end{array}$ & 0 & 0 & 0 & 0 & 1 & 0.8 & 119 & 99.2 \\
\hline $\begin{array}{l}\text { Consult the doctor when } \\
\text { changing the doses of medication }\end{array}$ & 0 & 0 & 0 & 0 & 1 & 0.8 & 119 & 99.2 \\
\hline $\begin{array}{l}\text { Put the immunosuppressive } \\
\text { medication in appropriate place }\end{array}$ & 0 & 0 & 0 & 0 & 1 & 0.8 & 119 & 99.2 \\
\hline Adherence score & \multicolumn{2}{|c|}{ Minimum } & & & & & & \\
\hline
\end{tabular}

Figure 1

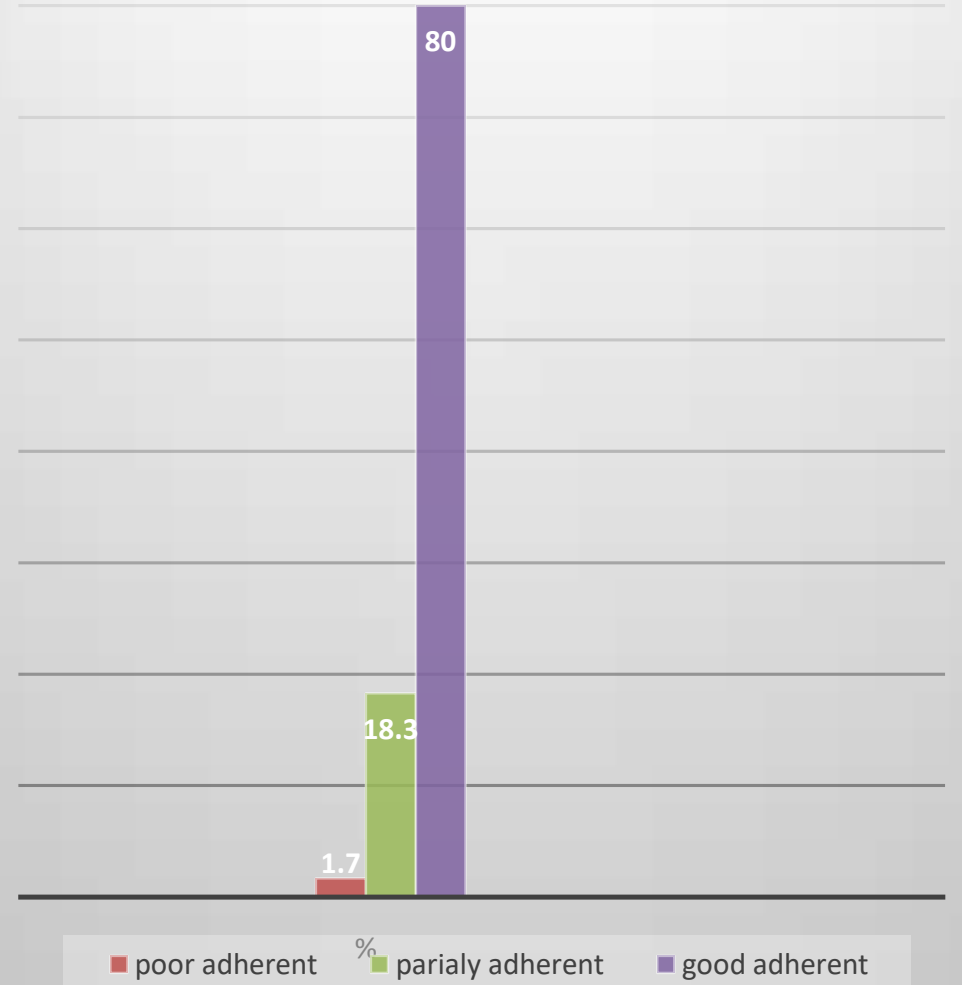

Figure (1): Total level of adherence to immunosuppressive medication among the renal transplant recipients $(n=120)$.

Table (9) levels of renal transplant recipients compliance $(\mathrm{n}=120)$ Level of compliance 
Lifestyle Modification Among Post Renal Transplant Recipients

\begin{tabular}{|c|c|c|c|c|c|c|c|c|c|c|c|}
\hline \multirow[t]{2}{*}{ Categories } & \multicolumn{2}{|c|}{ Non compliant } & \multicolumn{2}{|c|}{$\begin{array}{c}\text { Poor } \\
\text { compliant }\end{array}$} & \multicolumn{2}{|c|}{$\begin{array}{c}\text { Partial } \\
\text { compliant }\end{array}$} & \multicolumn{2}{|c|}{$\begin{array}{c}\text { Good } \\
\text { compliant }\end{array}$} & \multirow[t]{2}{*}{ Min } & \multirow[t]{2}{*}{$\operatorname{Max}$} & \multirow{2}{*}{$\underset{\mathbf{n}}{\text { Media }}$} \\
\hline & $\mathrm{NO}$ & $\%$ & $\mathrm{NO}$ & $\%$ & $\mathrm{NO}$ & $\%$ & $\mathrm{NO}$ & $\%$ & & & \\
\hline Medication & 1 & 0.8 & 1 & 0.8 & 71 & 59.2 & 47 & 39.2 & 15.0 & 30.0 & 28.0 \\
\hline $\begin{array}{c}\text { Dietary } \\
\text { recommendation }\end{array}$ & 4 & 3.3 & 23 & 19.2 & 65 & 54.2 & 28 & 23.3 & 12.0 & 30.0 & 27.0 \\
\hline $\begin{array}{c}\text { Fluid, body } \\
\text { temperature, and } \\
\text { daily weight }\end{array}$ & 5 & 4.2 & 24 & 20.0 & 82 & 68.3 & 9 & 7.5 & 10.0 & 27.0 & 20,0 \\
\hline Avoiding infection & 0 & 0 & 7 & 5.8 & 99 & 82.5 & 14 & 11.7 & 20.0 & 39.0 & 34.0 \\
\hline Follow up visits & 0 & 0 & 2 & 1.7 & 84 & 70.0 & 34 & 28.3 & 17.0 & 27.0 & 24.0 \\
\hline Social relation & 0 & 0 & 4 & 3.3 & 25 & 20.8 & 91 & 75.8 & 13.0 & 25.0 & 24.0 \\
\hline $\begin{array}{c}\text { Work post } \\
\text { transplantation }\end{array}$ & 16 & 13.3 & 21 & 17.5 & 23 & 19.2 & 60 & 50.0 & 0.0 & 9.0 & 8.5 \\
\hline Physical exercise & 72 & 60.0 & 19 & 15.8 & 24 & 20.0 & 5 & 4.2 & 0.0 & 12.0 & 6.0 \\
\hline Marital relation & 5 & 4.3 & 12 & 10.4 & - & - & 98 & 85.2 & 0.0 & 3.0 & 3.0 \\
\hline $\begin{array}{c}\text { Marital relation } \\
\text { for male }(n=67)\end{array}$ & 18 & 26.9 & 22 & 32.8 & 4 & 6.0 & 23 & 34.3 & 2.0 & 9.0 & 6.0 \\
\hline $\begin{array}{c}\text { Marital relation } \\
\text { For female }(\mathrm{n}= \\
\mathbf{3 3})\end{array}$ & 3 & 9.1 & 5 & 15.2 & 14 & 42.4 & 11 & 33.3 & 9.0 & 27.0 & 24.0 \\
\hline $\begin{array}{l}\text { Precaution of } \\
\text { Skin cancer }\end{array}$ & 91 & 75.8 & 23 & 19.2 & 4 & 3.3 & 2 & 1.7 & 0.0 & 12.0 & 5.0 \\
\hline $\begin{array}{c}\text { Precaution of } \\
\text { female breast } \\
\text { cancer }\end{array}$ & 34 & 89.5 & 4 & 10.5 & 0 & 0 & 0 & 0 & 0.0 & 9.0 & 3.0 \\
\hline Smoking & 55 & 45.8 & 0 & 0 & 0 & 0 & 65 & 54.2 & 6.0 & 12.0 & 12.0 \\
\hline Total all & 0 & 0 & 9 & 7.5 & 105 & 87.5 & 6 & 5.0 & 57.0 & 99.5 & 84.05 \\
\hline
\end{tabular}

$*$ Min $=$ minimum, Max $=$ maximum, Med $=$ median

Figure 2

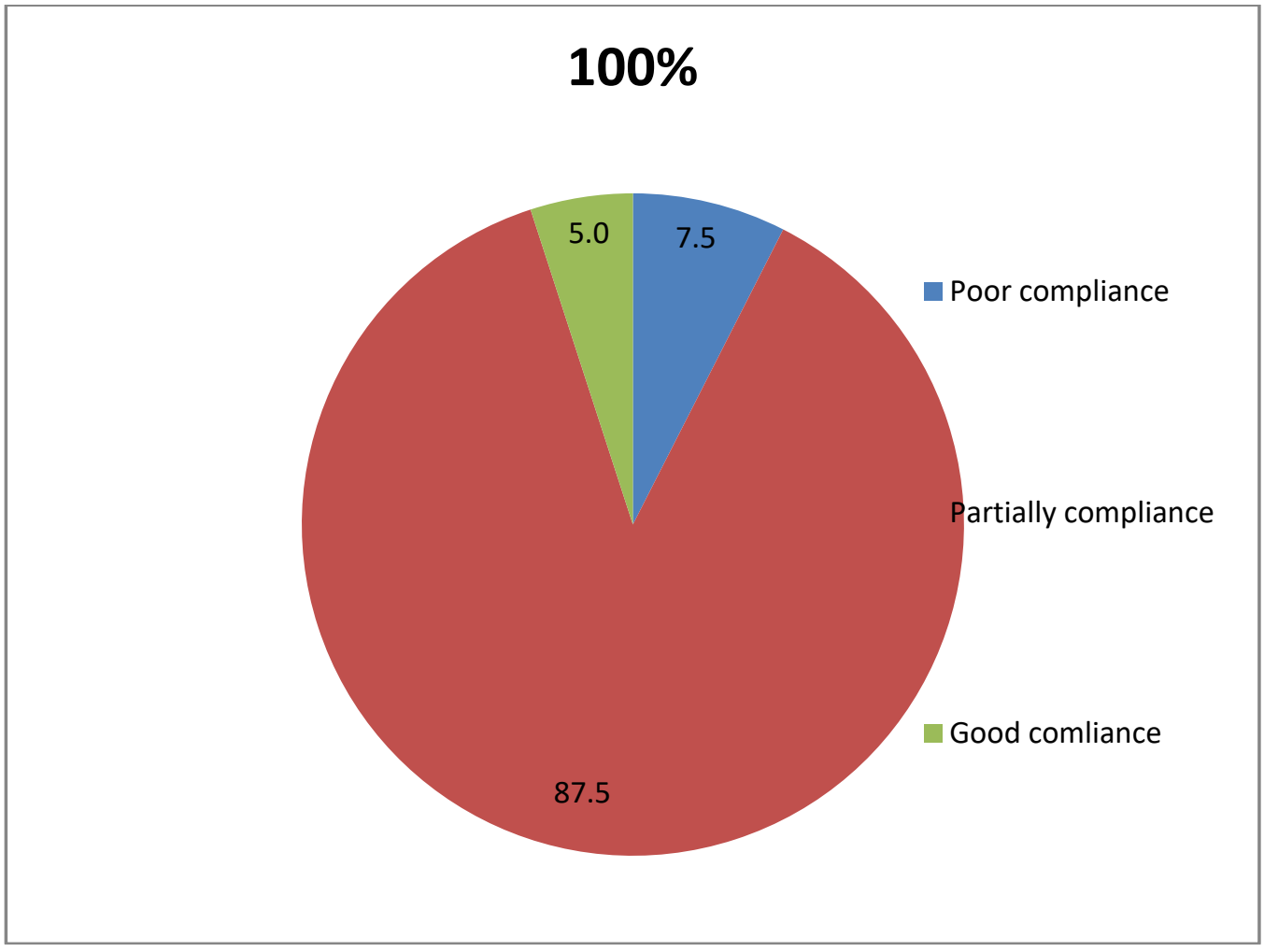

Figure (2) total level of compliance among post renal transplant recipients $(n=120)$.

Table (10) Association between socio demographic and economic characteristics of the renal transplant recipients and their total adherence score to immunosuppressive medication $(n=120)$.

\begin{tabular}{|l|l|l|l|l|l|l|}
\hline Socio demographic & No & minimum & maximum & median & Mean \pm SD & Significance test \\
\hline
\end{tabular}
characteristics 


\begin{tabular}{|c|c|c|c|c|c|c|}
\hline $\begin{array}{l}\text { Age group } \\
\begin{array}{l}18-28 \text { years } \\
28-38 \\
38-48 \\
48-58\end{array}\end{array}$ & $\begin{array}{l}66 \\
44 \\
8 \\
2 \\
\end{array}$ & $\begin{array}{l}34.0 \\
43.0 \\
48.0 \\
49.0\end{array}$ & $\begin{array}{l}51.0 \\
51.0 \\
51.0 \\
50.0\end{array}$ & $\begin{array}{l}50.0 \\
51.0 \\
51.0 \\
49.0\end{array}$ & $\begin{array}{l}49.24 \pm 3.87 \\
49.73 \pm 2.02 \\
50.25 \pm 1.39 \\
49.50 \pm 0.71\end{array}$ & $\begin{array}{l}F=0.484 \\
p \quad 0.694\end{array}$ \\
\hline $\begin{array}{l}\text { Sex } \\
\text { Male } \\
\text { Female } \\
\end{array}$ & $\begin{array}{l}82 \\
38 \\
\end{array}$ & $\begin{array}{l}35.0 \\
34.0 \\
\end{array}$ & $\begin{array}{l}51.0 \\
51.0 \\
\end{array}$ & $\begin{array}{l}51.0 \\
50.0 \\
\end{array}$ & $\begin{array}{l}49.88 \pm 2.17 \\
48.66 \pm 3.61 \\
\end{array}$ & $\begin{array}{l}t=2.299 * * \\
\mathrm{p} 0.023\end{array}$ \\
\hline $\begin{array}{l}\text { Marital status } \\
\text { Married } \\
\text { Divorce } \\
\text { Single }\end{array}$ & $\begin{array}{l}71 \\
3 \\
46\end{array}$ & $\begin{array}{l}34.0 \\
43.0 \\
35.0\end{array}$ & $\begin{array}{l}51.0 \\
47.0 \\
51.0\end{array}$ & $\begin{array}{l}51.0 \\
45.0 \\
50.0\end{array}$ & $\begin{array}{l}49.4 \pm 2.34 \\
45.0 \pm 2.00 \\
49.15 \pm 3.09\end{array}$ & $\begin{array}{l}\mathrm{F}=5.518 * * \\
\mathrm{p} 0.005\end{array}$ \\
\hline $\begin{array}{l}\text { Education } \\
\text { Illiterate } \\
\text { Read and write } \\
\text { Secondary } \\
\text { University }\end{array}$ & $\begin{array}{l}9 \\
18 \\
66 \\
27\end{array}$ & $\begin{array}{l}48.0 \\
34.0 \\
43.0 \\
35.0\end{array}$ & $\begin{array}{l}51.0 \\
51.0 \\
50.0 \\
51.0\end{array}$ & $\begin{array}{l}51.0 \\
51.0 \\
50.0 \\
51.0\end{array}$ & $\begin{array}{l}50.11 \pm 1.17 \\
48.78 \pm 4.65 \\
49.59 \pm 1.91 \\
49.51 \pm 3.24\end{array}$ & $\begin{array}{l}F=0.579 \\
p 0.630\end{array}$ \\
\hline $\begin{array}{l}\text { Occupation } \\
\text { Don't work } \\
\text { Trade work } \\
\text { Administrative } \\
\text { Professional } \\
\text { Manual work }\end{array}$ & $\begin{array}{l}44 \\
16 \\
31 \\
23 \\
6\end{array}$ & $\begin{array}{l}34.0 \\
43.0 \\
48.0 \\
46.0 \\
47.0 \\
\end{array}$ & $\begin{array}{l}51.0 \\
51.0 \\
51.0 \\
51.0 \\
51.0\end{array}$ & $\begin{array}{l}50.0 \\
49.5 \\
51.0 \\
51.0 \\
51.0\end{array}$ & $\begin{array}{l}48.59 \pm 3.97 \\
49.25 \pm 2.21 \\
50.13 \pm 1.06 \\
50.39 \pm 1.23 \\
50.17 \pm 1.60\end{array}$ & $\begin{array}{l}\mathrm{F}=2.509 * * \\
\mathrm{p} 0.046\end{array}$ \\
\hline $\begin{array}{r}\text { Residence } \\
\text { Urban } \\
\text { Rural }\end{array}$ & $\begin{array}{l}20 \\
100\end{array}$ & $\begin{array}{l}43.0 \\
34.0\end{array}$ & $\begin{array}{l}51.0 \\
51.0\end{array}$ & $\begin{array}{l}50.5 \\
51.0\end{array}$ & $\begin{array}{l}49.70 \pm 2.0 \\
49.45 \pm 7.88\end{array}$ & $\begin{array}{l}t=0.369 \\
p 0.713\end{array}$ \\
\hline $\begin{array}{c}\text { Monthly income } \\
1000->1500 \\
1500->2000 \\
\geq 2000\end{array}$ & $\begin{array}{l}43 \\
64 \\
13\end{array}$ & $\begin{array}{l}34.0 \\
35.0 \\
49.0\end{array}$ & $\begin{array}{l}51.0 \\
51.0 \\
51.0\end{array}$ & $\begin{array}{l}50.0 \\
51.0 \\
51.0\end{array}$ & $\begin{array}{l}48.91 \pm 3.38 \\
49.64 \pm 2.46 \\
50.69 \pm 0.63\end{array}$ & $\begin{array}{l}F=2.352 \\
p 0.100\end{array}$ \\
\hline
\end{tabular}

** There was statistically significance association

Table (11) Association between socio-demographic and economic characteristics of renal transplant recipients and their total compliance score $(\mathrm{n}=120)$

\begin{tabular}{|c|c|c|c|c|c|c|}
\hline $\begin{array}{l}\text { Socio demographic } \\
\text { characteristics }\end{array}$ & No & minimum & maximum & Median & Mean \pm SD & Significance test \\
\hline $\begin{array}{cr}\text { Age } & \text { group } \\
18-28 & \text { years } \\
28-38 & \text { years } \\
38-48 & \text { years } \\
48-58 & \text { years }\end{array}$ & $\begin{array}{l}66 \\
44 \\
8 \\
2\end{array}$ & $\begin{array}{l}57.0 \\
65.6 \\
75.0 \\
72.2\end{array}$ & $\begin{array}{l}98.0 \\
99.5 \\
90.0 \\
82.6\end{array}$ & $\begin{array}{c}82.6 \\
86.45 \\
83.50 \\
77.40\end{array}$ & $\begin{array}{l}81.67 \pm 8.40 \\
86.13 \pm 7.48 \\
83.46 \pm 4.79 \\
77.40 \pm 5.20\end{array}$ & $\begin{array}{c}\mathrm{F}=3.204 * * \\
\mathrm{p} \quad 0.026\end{array}$ \\
\hline $\begin{array}{l}\text { Sex } \\
\text { Male } \\
\text { Female }\end{array}$ & $\begin{array}{l}82 \\
38 \\
\end{array}$ & $\begin{array}{l}63.0 \\
57.0 \\
\end{array}$ & $\begin{array}{l}98.0 \\
99.5 \\
\end{array}$ & $\begin{array}{c}83.15 \\
86.8 \\
\end{array}$ & $\begin{array}{l}82.63 \pm 7.28 \\
48.91 \pm 9.57 \\
\end{array}$ & $\begin{array}{l}\mathrm{T}=1.437 \\
\mathrm{p} \quad 0.153\end{array}$ \\
\hline $\begin{array}{l}\text { Marital status } \\
\text { Married } \\
\text { Divorce } \\
\text { Single }\end{array}$ & $\begin{array}{c}71 \\
3 \\
46\end{array}$ & $\begin{array}{l}65.6 \\
77.3 \\
57.0\end{array}$ & $\begin{array}{l}99.5 \\
91.0 \\
96.0\end{array}$ & $\begin{array}{l}87.0 \\
79.0 \\
79.0\end{array}$ & $\begin{array}{l}86.28 \pm 6.82 \\
82.43 \pm 7.47 \\
78.91 \pm 8.06\end{array}$ & $\begin{array}{c}\mathrm{F}=14.140^{* *} * \\
\mathrm{p} \quad 0.000\end{array}$ \\
\hline $\begin{array}{l}\text { Education } \\
\text { Illiterate } \\
\text { Read and write } \\
\text { Secondary } \\
\text { University }\end{array}$ & $\begin{array}{c}9 \\
18 \\
66 \\
27\end{array}$ & $\begin{array}{l}77.0 \\
57.0 \\
63.0 \\
65.6\end{array}$ & $\begin{array}{l}92.7 \\
98.0 \\
99.5 \\
97.0\end{array}$ & $\begin{array}{l}85.9 \\
81.4 \\
83.5 \\
87.6\end{array}$ & $\begin{array}{l}84.88 \pm 5.04 \\
81.83 \pm 9.25 \\
82.68 \pm 7.91 \\
85.53 \pm 8.48\end{array}$ & $\begin{array}{l}F=1.126 \\
p 0.342\end{array}$ \\
\hline $\begin{array}{l}\text { Occupation } \\
\text { Don't } \\
\text { Trade } \\
\text { Administrative } \\
\text { Professional } \\
\text { work }\end{array}$ & $\begin{array}{c}44 \\
16 \\
31 \\
23 \\
6\end{array}$ & $\begin{array}{l}57.0 \\
68.0 \\
63.0 \\
65.0 \\
68.0\end{array}$ & $\begin{array}{l}99.1 \\
94.0 \\
99.5 \\
97.0 \\
95.3\end{array}$ & $\begin{array}{l}81.85 \\
81.05 \\
85.00 \\
88.00 \\
83.80\end{array}$ & $\begin{array}{l}81.83 \pm 8.10 \\
81.80 \pm 7.34 \\
85.05 \pm 7.83 \\
85.36 \pm 8.51 \\
82.30 \pm 9.17\end{array}$ & $\begin{array}{c}\mathrm{F}=1.264 * * \\
\mathrm{p} 0.288\end{array}$ \\
\hline $\begin{array}{l}\text { Residence } \\
\text { Urban } \\
\text { Rural }\end{array}$ & $\begin{array}{c}20 \\
100 \\
\end{array}$ & $\begin{array}{l}63.0 \\
57.0 \\
\end{array}$ & $\begin{array}{l}99.5 \\
99.1 \\
\end{array}$ & $\begin{array}{c}87.0 \\
83.85 \\
\end{array}$ & $\begin{array}{l}84.61 \pm 9.81 \\
83.11 \pm 7.75 \\
\end{array}$ & $\begin{array}{c}T=0.755^{* *} \\
\text { p } 0.425\end{array}$ \\
\hline $\begin{array}{l}\text { Monthly } \\
1000->1500 \\
1500->2000 \\
\geq 2000\end{array}$ & $\begin{array}{l}43 \\
64 \\
13\end{array}$ & $\begin{array}{l}57.0 \\
63.0 \\
65.6\end{array}$ & $\begin{array}{l}99.1 \\
99.5 \\
97.0\end{array}$ & $\begin{array}{c}80.4 \\
84.55 \\
89.00\end{array}$ & $\begin{array}{l}80.76 \pm 83.30 \\
84.32 \pm 7.30 \\
87.20 \pm 9.21\end{array}$ & $\begin{array}{l}\mathrm{F}=4.345^{* *} \\
\mathrm{p} 0.015\end{array}$ \\
\hline
\end{tabular}

** There was statistically significance association

Figure (3): Correlation between kidney transplant recipients' adherence and their compliance $(n=120)$ 


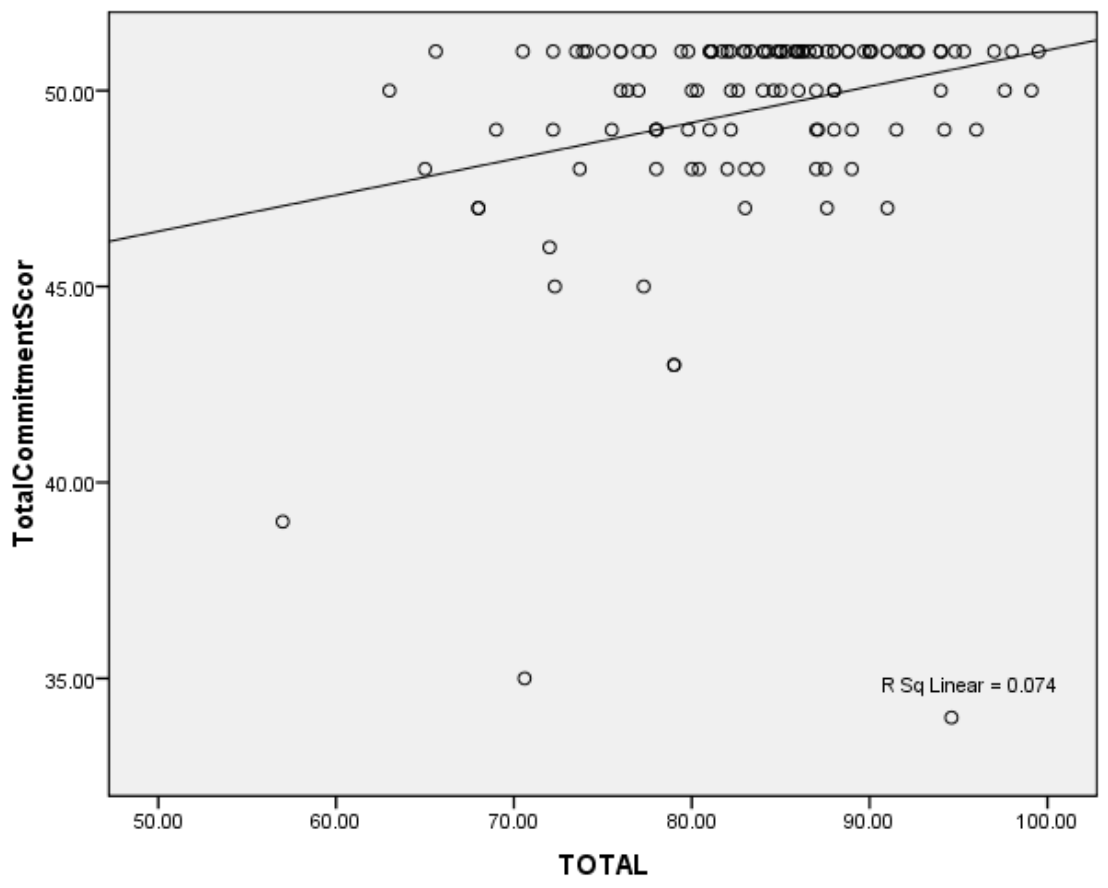

Figure (3) Illustrates significant correlation between kidney transplant recipients, adherence and their compliance, $(\mathrm{r}=+0.272 \& \mathrm{p}=0.003)$

\section{Discussion}

Successful renal transplantation enables the recipient to increase kidney survival and improve quality of life through freedom from dialysis, fluid restriction and increase exercise capacity, sexual function and fertility, improve overall sense of wellbeing it, also less expensive than dialysis and better for the health economics (Steddon, et al., 2014). Lifestyle is the manner of living including behavior that promotes or impairs good health and longevity, lifestyle guide may affect health positively or negatively. The patient post renal transplantation is feeling better and looking forward to return to his normal lifestyle (Urstad, 2013).

Compliance of renal transplant patients with their recommended lifestyle behaviors is functional for two reasons: first, it may be argued that it is unwanted to transplant an organ into a patient who is flat to serious noncompliance pre-transplantation. Second, identifying such high-risk patients allows supportive patient education to be designed, which optimizes self-care, and hopefully, promotes compliance (Ghieth, et al., 2008).

Renal transplant recipients were at high risk to developed un wanted weight gain, hyperglycemia, hypertension, osteoporosis and muscle atrophy, so renal transplant recipients are required to compliance with recommended lifestyle behaviors as regular physical exercise, beneficial eating habits and calorie intake, follow up care, skin cancer precautions, correct sun protection, skin self care examination especially for those who are planning a warm weather vacation or exposure to the sun during the summer (Chapman 2010, Neyhart, 2009 \& Fuerstein, \& Geller, 2008). As well renal transplant recipients are required to take lifelong immunosuppressive medication to prevent graft rejection. Non-adherence to immunosuppressive medication is a common issue and increases over time. Both dosage and timing of medication are crucial. Failure to take the medication as prescribed is a risk factor for (early) acute rejection or (late) graft failure/loss, and patient mortality (Tielen, et al., 2014).

In relation to socio-demographic and economical characteristic of the renal transplant recipients their age were 18-58 years, more than half of them aged from 18-28 years with mean ${ }^{ \pm}$SD age of $34.11 \pm 7.08$ years. The result of present study agreed with Omar, (2009), who mentioned that his subjects were aged from 8-

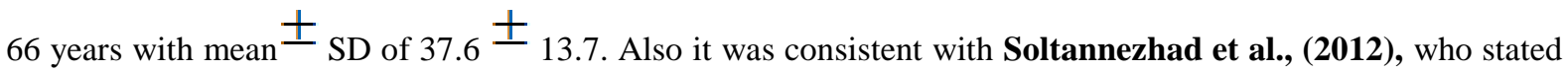
that mean age of their subjects was $37.88 \pm$ 9.72, and with Baker and Ghoniem (2005), who stated that their sample age ranged from 12-30 years.

The results of present study were contrary with Tielen et al., (2013), who found that their study sample ranged from 19 -75 years, and Tayebi et al., (2010) who found that their study sample aged from 
15-75 years old. A cording to Steddon et al., (2014), renal transplantation didn't carry out for patients whose age of 60 as the commonest of graft loss beyond this age and death with functioning graft, Urology and Nephrology Center affiliated to Mansoura University follow these regulations.

As regards to gender, the present study showed that more than two thirds of the study sample was male this finding was supported by Ghieth et al., (2008), and Bautista, et al., (2007), who mentioned that male had a significantly higher rate than female, and Ramadan, et al0, (2013), who reported that more than three quarters of their subjects were male, in addition to Tayebi et al., (2010), who stated that more than half of their sample was male. While it was contrary with Kosaka, et al., (2013), who stated that two thirds of their sample was female. In relation to marital status, the current study showed that more than half of the study sample was married. This was consistent with Khattak, et al., (2010), who reported that the majority of their studied subjects were married, and supported by Ramdan et al., (2013), who reported that more than half of their patients were married. This may be related to most study subjects were aged 18-38 years and this usually age of marriage according to the Egyptian culture farther more the renal transplant recipients were looking forward to return to normal life. Concerning educational level the results of this study illustrated that more than half of the study sample was secondary school degree. This finding was consistent with Soltannezhad, et al., (2013), they mentioned that more than three fourths of their sample had high school diploma or lower education. While the finding of the present study was contrary with Rumyantzeu, et al., (2006), who found that the majority of their subjects had a high degree of educational level, and Ghieth et al., (2008), they stated that most of their study sample was highly educated. These differences may be due the majority of Egyptians' belonged to middle level of education.

In relation to occupation the current study demonstrated that almost two thirds of the study sample was working and this was consistent with Kuiper, (2011), who found that the majority of his subjects were working, but was contrary with El-Manzalawy et al., (2006), who found that two thirds of their subjects were not working, it also was disagreement with Kosaka, et al., (2013), who found that less than two thirds of their sample was not working; this could be related to wail by others or presence of social security. Concerning residence, the result of current study revealed that, the majority of the study sample was living at rural area. This finding supported by El saadney et al., (2008), who reported that the majority of their sample was from rural area but it was contrary with Mandor, (2013) and El-manzalawy, et al., (2006), who reported that more than half of their subjects were from urban area. This may be due to most of attendants to Urology and Nephrology Center belonged to Delta region. As regard to renal transplant recipients health profile; the current study illustrated that the majority of the study sample had unknown cause of renal failure and minority had hypertension as cause of renal failure this was consistent with Mandor, et al., (2013), who stated that more than half of group one were had unknown cause of renal failure and less than half of group two had hypertension while this was contradicted with National Kidney Foundation, (2012), which reported that high blood pressure is the most common cause of renal failure and Kosaka, et al., (2013), who stated that one fourths of their subjects were hypertension.

Also the current study illustrated that more than three fourths of the study sample was on hemodialysis before transplantation and this result was consistent with Kosaka, et al., (2013), who stated that the majority of their subjects were on hemodialysis before renal transplantation, also it supported by Ramadan, (2013) and Mandor, (2013), who stated that the majority of their sample was on hemodialysis pre renal transplantation. The current study showed that the majority of the study sample received the kidney from related living donor this was consistent with Mandor, (2013), who stated that the majority of his groups received kidney from related donor also this finding agreed with Kosaka, (2013) and Ramadan, et al., (2013), who reported that more than three quarter of their subjects were first degree related donor. This could be attributed to availability of cadaveric donor wasn't applied in Egypt, in addition to renal transplantation is most successful when kidney came from a related lived donor and it is the legal option for Egyptians.

Regard to post renal transplantation complications; the current study illustrated that more than one fourth and minority of the study sample was hypertensive and diabetic respectively, this finding consistent with Mandor, (2013 and Jimeneze, et al., (2009), who reported that the minority of their finding had hypertension and diabetes post transplantation. This may be due to side effects of immunosuppressant drugs post renal transplantation. Regarding rejection post renal transplantation this study found that minority of the study sample had acute rejection. This was consistent with ELsaadany, (2008), who stated that one fourth of his sample had acute rejection. Danovitch, (2012) stated that rejection is one of the major complication which face renal transplant recipient especially early post renal transplantation. Regarding drug used post renal transplantation, the current study found that all study samples received immunosuppressive medication, vitamins and minerals, these findings were consistent with EL Saadany, (2008), who stated that all their subjects receive immunosuppressive medication, vitamin and minerals. According to National kidney foundation, (2012), renal transplant recipients must receive immunosuppressive medication, minerals vitamins and anti acids to prevent complication related immunosuppressant, and increase graft survival. 
Concerning physical examination, the current study found that more than one fourths of the study sample had mild hypertension 140/100. This was consistent with Kosaka, et al., (2013), who found that more than one fourth of the study sample was hypertension. It was consistent with Joho, (2012), who found that more than half was hypertensive and their blood pressure was average $140 \mathrm{mmhg}$ or greater. Diastolic blood pressure $90 \mathrm{~mm}$ hg or greater, this was secondary to dyslipidemia or salt dietary habits in renal transplant recipients. Regarding laboratory investigations (CBC, Kidney function test, liver function test, and FK level), the current study found that all the study sample had normal finding. This finding was consistent with ELSaadeny, (2008), who stated that all of their finding had laboratory investigations with normal ranges. KDIGO, (2009) found that to maintain good health, increase kidney survival, and provide high quality of life renal transplant recipient must be assessed frequently by laboratory investigation and it must be in normal ranges.

Regarding urine output the current study found that two thirds of the study sample was excreted 1.5-3 liters per day which equal $0.5 \mathrm{ml} / \mathrm{kg} / \mathrm{h}$, all of them drank $(1.5 \geq 2.5)$ litters per day, and the majority of them preferred pure water. According to Anita and Dodidin, (2012) and Mclntosh, ( 2015) stated that an adequate fluid intake for adult men is approximately 3 liters a day and 2.2 liters for adult women and pure water is the best and excellent beverage for renal disease and kidney transplant recipient. Concerning BMI, the current study found that the majority of the study sample was overweight more than $30 \mathrm{Kg} / \mathrm{m}^{2}$. This was agreement with Demuster, (2007) and Lkizer, (2012), who stated that post renal transplantation mild obesity was in less than two thirds of the study sample. On the opposite side, it was not consisted with Abraham, et al., (2014), who stated that less than two thirds of their study sample was mild obesity $30-34.99 \mathrm{Kg} / \mathrm{m}^{2}$. This may be related to sedentary lifestyle and lake of knowledge. The extremes of very high and very low BMI and increase appetite which face renal transplant recipients and genetic factor before renal transplantation are important risk factor affecting patient and graft survival.

Regarding side effects related to immunosuppressive medication which face renal transplant recipients the current study found that less than two thirds of the study sample complained from those side effects, only one eight of them had skin papule and minority had vision diminution. Mandel, (2016) and Danovitch, (2012) stated that renal transplant recipient may had some manifestation related to immunosuppressive medication as hairsuitism, face edema, cataract, skin papule, and vision diminution, with long term usage of immunosuppressive medication. As well Mories, (2008) and Baker, (2014) stated that skin papule, and vision diminution were side effects which face renal transplant recipient related to long term immunosuppressive medication. Good nutrition post renal transplantation is an important factor and plays a key role in renal transplant recipient's lifestyle (National Kidney Foundation 2012). The current study explained that the majority of the study sample had three meals per day. Colmpia University, (2016) advice renal transplant recipient to had three regulatory meals per day, also Guys, and Thoms, (2014) stated that renal transplant recipient need an essential three meals per day breakfast, lunch and dinner at fixed times every day. The majority of the current study sample had fried, salted, sugary, and use animal oil which prevented post renal transplantation. National Kidney Foundation, (2012) and Colombia University, (2013) advice renal transplant recipient to restrict in salted, sugary, fried and use platy oil to prevent obesity and new onset diabetes post renal transplantation.

Regarding tea and coffee consumption post renal transplantation the current study found that minority of the study sample drank 3 cups of tea and minority drank 3 cups coffee. Steddon, et al., (2014) advice renal transplant recipient to restrict in drinking tea and coffee as more than 5 cups of coffee per day increase systolic blood pressure 10-15 mm hg for several hours, also Mclntosh, (2015) stated that beverage containing caffeine not ideal as it having diuretic properties which cause the body release water. Concerning smoking post renal transplantation the current study found that only one eight of the study sample had cigarette smoking post renal transplantation. This was agreed with Nourbal, et al., (2011), they found that more than one third of renal transplant recipients were smoke at the time of transplantation and about one eight only continued smoking post renal transplantation, this was the opposite with Silva et al., (2016), who stated that more than one third were smoker post renal transplantation. These differences due to different perception of post renal transplant recipients to smoke. This may be due to smoking prevalence among renal transplant recipients is more difficult to estimate and varies greatly across studies.

As regard sleeping post renal transplantation the current study showed that majority of the study sample was sleep well and sleep on graft site post renal transplantation. EL Saadney, (2008) stated that renal transplant recipients sleep well and sleep on the site of graft, while. This was the opposite with Bukhalter, et al., (2013), who stated that half of their subjects had poor sleeping post renal transplantation. It might be related to social, psychological problem, fear, anxiety or may related increasing sleeping hours in day time.

Concerning stress management post renal transplantation, the current study found that the study sample managed stress by different methods through relative visits, reading Qur'an, sleep, or practice physical activity, and sports. This was agreement with Kosaka, et al., (2013), who stated that the majority of their subjects were receive sufficient support from their family and friends to manage their stress. Stedden, et al., (2014) stated that 
renal transplant recipients should had cognitive therapy and modest effect. As regard to physical activity the current study stated that more than one third of the study sample practice physical activity; three fourths of them walk half an hour daily, while one fourth walk an hour daily. This was agreement with Nielens et al., (2010), they found in their study that the behavior of physical activity in advanced phase of renal transplantation is unclear. KDIGO, (2012) and WHO, (2012) recommended that renal transplant recipients to practice at least 30 min of moderate intensity physical activity as walking every day.

Finally, very limited data suggest the hypothesis of link between physical activity and clinical outcome but no evidence on the impact of physical activity and exercise training on morbidity and mortality and graft survival in kidney transplant recipients. As a whole up till now it seems judicious to counsel and encourage for more physical activity as a part of routine medical care in renal transplantation (Bellizzi, et al., 2014). Concerning reproductive and sexual health the current study found that more than three fourths of the study sample counseled before married. National kidney Foundation, (2014) advice kidney transplant recipients to counsel and ask doctors about the probate time for marriage, and wait until the scare has begun to heal, and your doctor says it is all right to resume sexual activity and there is no reason to worry about the graft kidney.

In relation to return of female menstrual cycle the current study found that less than two third of female had rturn menstruation with first month after renal transplantation. This was agreement with (Ghieth et al., 2008), who found that more than half of their subjects had return menstruation within the first months post renal transplantation. Pezeshki, (2013) and Danovitch, (2012) stated that fertility is usually restated in women with in first few months post renal transplantation.

Concerning men problem related to $s$ xual function the current study found that the majority of the study smple was not face any sexual problem, and all of them were consult doctors. This finding consisted with Gieth, et al., (2008), who found that more than half of their study sample was not suffer from any sexual dysfunction. Danovich, (2012) reported that renal transplantation improve fertility post renal transplantation in both male and female. As regard to number of children pre-and post-renal transplantation between male and female. The current study found that number of children between male recipients was equal pre-and post transplantation while in female recipients' number of children pre-transplantation was more than post transplantation. Gieth, et al., (2008) and Pezeshki, (2013) stated that number of children was equal between male and female recipients pre-and post-renal transplantation. Also, Morise, (2008) and Pezeshki, (2013) stated that pregnancy and child peering was better post renal transplantation among female recipients' comparison to dialysis. This difference might be due to female age and family size and female don't need children in this stage of their life. As regard to problem which facing women related to sexual activity, the current study stated that more than one third of them had urinary tract infection. Pezeshki, (2013) stated that urinary tract infection was the most common problem which faces women in child peering period especially bacterial infection which appear in less than one third of women in child peering period. Concerning contraceptive method usage post renal transplantation, the current study found that less than two thirds use local or oral contraceptive method. Pezeshki, (2013) showed that usage low dose of estrogen, progesterone oral contraceptive post renal transplantation was advised.

Post renal transplant recipients' adherence to immunosuppressive medication. The current study explained total level of adherence among renal transplant recipients post renal transplantation, found that the majority of study sample had a good adherence to immunosuppressive medication this finding consisted with Tielen, et al., (2014), who stated that the majority of their subjects had adhered to immunosuppressive medication. Also, was agreement with Kobus et al., (2011) who found that the most of renal transplant recipients had a good adherent with immunosuppressive medication. These findings were contrast with Chisholm, (2004), who found that approximately half of their subjects were non-adherent to immunosuppressive medication. Renal transplant recipients' non-adherent classified in many forms the common form corresponding in missing doses. The current study found that minority of the study sample had missing dose. This consisted with Danovitch, (2012), who surveyed less than one fourth of the study sample was admitted missing doses of immunosuppressive medication. It also was agreement with Tielen, et al., (2013) who stated that minority of their sample had missed dose. As regard to stop immunosuppressive medication the current study found that none of the study sample had stopped or underestimate the prescribed dose. This agreed with Tielen, et al., (2013), who stated that none of their subjects had under estimate or completely stopped the prescribed medication. This due to renal transplant recipients feared from graft loss or return to dialysis.

The current study found that most of the study sample checked the level of immunosuppressive medication after 12 hour of the past dose every visit. Backer, (2014) stated that renal transplant recipient must measure prografe, nural and imuran level frequently to estimate the accurate dose and prevent medication toxicity. The policy of Urology and Nephrology Center affiliated to Mansoura University impose renal transplant recipients to measure the medication level and supply immunosuppressive medication for all renal transplant recipients every visit. The current study found that most of the study sample had a good adherence and knowledgeable about medication name, form, color, and prescribed dose. These findings consisted with 
Omar, (2009), who found that more than half of their subjects had enough knowledge about immunosuppressive medication as a part of post renal transplantation care.

As regard to compliance of renal transplant recipients regarding medication, dietary recommendation, follow up of fluid, body temperature, and daily weight, avoid infection, follow up visit, social relation, work, practicing daily physical exercise, marital relation, marital relation for male, marital relation for female, precaution of skin cancer, and female breast cancer, and smoking post renal transplantation.

The current study found that the majority of the study sample had a partial level of compliance, this finding was consistent with Omar, (2009), who found that more than three quarters of his subjects were had good practices in relation to post renal transplantation care. The current study found that more than half of the study sample was partial compliant regard other medication rather than immune-suppressive medication. This result consisted with Geith, et al., (2008), who found than more than three fourths of their study subjects were compliant with medication other than immunosuppressant. The current study found that one fourth of the study sample had good compliance regard nutrition, this finding agreed with Mandore, (2013), who found that more than one fifth in group 2 and minority in group 3 had compliance with dietary regimen post renal transplantation. It also agreed with Kobus, et al., (2011), who administrated that majority of renal transplant recipients failed to fulfill compliance with dietary recommendation.

The current study found that the majority of renal transplant recipient had compliant with follow up of fluid, body temperature, blood pressure, and daily weigh. Chitra, (2013) mentioned that fluid, and electrolyte requirements should be evaluated daily, also hydration must be monitored closely post renal transplantation.

Infection was the most common problem which faces renal transplant recipients post renal transplantation. the current study found that most of the study subjects had compliant with avoiding infection post renal transplantation. This finding consisted with Mandor., (2013), Kasiske., et al., (2009) and Geith, et al., (2008) who found that majority of their sample size had a good compliant with infection control measure as daily bath in summer, avoid infected person, teeth brushing and avoid contact with immunized infant. he urrent study found that less than three fourths of the study sample was partial compliant with follow up schedule post renal transplantation this finding supported by Omar, (2009) and Mandor, (2013), who stated that most of their subjects had compliant with follow up visits post renal transplantation. This was due to Urology and Nephrology Center affiliated to Mansoura University provide total medical health care in addition to for free medication. The current study showed that more than three fourths of the study sample had a good compliant toward social activities. This finding supported by Joshi et al.., (2013), who stated that renal transplant recipient was socially better post renal transplantation compared with End Stage Kidney Disease (ESKD). This finding also agreement with Depasquale, et al., (2014), who stated that living donor especially related donor provide a good family support, improve social relation and help kidney transplant recipient to overcome problem and care of graft kidney beside that improve relationship with donor from $86 \%-100 \%$.

This finding also supported by Danovitch, (2012), who mentioned that majority of renal transplant recipients had compliant with social relation and functioning psycho-socially at normal levels compared with dialysis and ESKD. This was due to successful renal transplant recipients free from dialysis sessions which necessity three days for four hours, saving this time for social activities. The current study found that half of the study sample had a good compliant related to work post renal transplantation. National Kidney foundation, (2014) stated that renal transplant recipients can return to work, school and university post renal transplantation when they physically, mentally and socially normal. Ass well Danovitch, (2012), Mcpake and Bumapp., (2009) stated that renal transplant recipients can return to work within 2 months and can drive inside the country from 2-3 months post renal transplantation when they were physically normal and after nephrologists' consolation. Concerning physical exercise post renal transplantation, the current study found that less than two thirds of the study sample was non-compliant toward exercise. This finding supported by Gieth, et al., (2008), who stated that more than one third of the study subjects had noncompliant to physical exercise (walk). This finding contrast with Mandor, (2013), who mentioned that majority of their subjects had compliant to exercise regimen and participating sports. It also contrasts with (Kobus, et al., 2011), who found that most of their subject reported various form of physical activity. This might may be due to lake of knowledge about the importance of exercise and absence of proper places for exercise.

Concerning marital relation for male, the current study found that more than one third of the study sample had a good compliance with marital relation. This finding supported by Schmidt, et al., (2007), who mentioned that two third of men experience improved libido and sexual function. Ptel and lee., (2012) mentioned that renal transplantation improve overall health and improve sexual functioning, they advised kidney transplant recipient to ask about their sexual function, and referred to urologist as necessary.

For female compliance concerning marital relation post renal transplantation, the current study found that one third of renal transplant recipients had a good compliance with marital relation this finding supported by Mandor, (2013) and Gieth, et al., (2008), who stated that all of the married female had a good compliance 
with marital relation post renal transplantation. Regarding precaution of skin cancer, the current study found that more than three fourths of renal transplant recipients had a none compliance concerning precaution of skin cancer, this finding consisted with Mandor, (2013), who mentioned that most of their subjects had noncompliant concerning skin cancer precaution. This finding contrast with Vanacker and Maes, (2012), who mentioned that renal transplant recipients avoid exposure to solar ultraviolet radiation, sun exposure (especially during a peak hour of radiation), use of effective sun screen, wear protective cloths and annual skin examination by a dermatologist is recommended especially for high risk patient as who living in high sun exposure climate. The difference may come from that the current study sample unknowledgeable concerning predisposing factors develop skin cancer post renal transplantation.

Concerning precaution of female breast cancer the current study found that the majority of them had noncompliant concerning breast cancer precautions. This finding supported by Mandor, (2013), who stated that all of their subjects were none adherent with breast self examination and mammogram after age of 40 . Vanacker and Maes, (2012) mention that renal transplant female were advised to make regular self examination to breast, notify physician when notice any change, and make mammogram after age of 40 . The concept of early detection of breast cancer is not with instilled among Egyptian women. Concerning smoking post renal transplantation the current study found that more than half of the study sample had a good compliant concerning smoking post renal transplantation this finding supported by Mandor, (2013), who stated that majority in group1, less than three fourths in group 3 were adhered to non and stop smoking post renal transplantation.

Association between socio-demographic and economic characteristics of renal transplant recipients is their total adherence score to immunosuppressive medication.

The current study illustrated that there was statistically significant association between sex, marital status and occupation of renal transplant recipients, and their total adherence score to immunosuppressive medication this was supported by Morise, (2008), who mention that sex affect adherence, female sex was defined as a non-adherence toward immunosuppressive medication. It also was agreement with Prendergast and Gaston, (2010) and Denhaernck, et al., (2007), who mention that gender had significant effect on patient adherence post renal transplantation but male gender defined as none adherence. These differences may be due to male renal transplant recipients had more concern about their health than female and their health had first priorities in their daily life or may be that male number in current study more than female. Related to marital relation the current study finding consistent with Weng, et al., (2013), who stated that renal transplant recipients who lived alone or received low social support was develop a none adherence and Griva, et al., (2012), who mentioned that marital status had a significant association on recipients' adherence toward immunosuppressive medication post renal transplantation as non-adherence present between married or whom had cohabiting relationship.

Concerned with occupation the result of the current study agreed with Griva., (2012), who mentioned that work status had a significant effect on patient adherence toward immunosuppressive medication but employed renal transplant recipients were non adherent because they were occupied and always forget to take immunosuppressive medication, while it contradicted with Prendergast et al., (2010), who found that employment status was associated with none adherence to immunosuppressive medication as they were at high risk to perceived stress which lead to none adherence. This difference may be due to the different nature of work.

Concerning association with age, educational level, residence, and monthly income of renal transplant recipients there was no statistically significant association with those variables and their total adherence to immunosuppressive medication. Related to age, this finding was consistent with Tielen, et al., (2014), who found that age had no significant difference toward patients' adherence. This was opposite to Folkman, et al., (2008) and Van, et al., (2007), who found that age had a significantly affect patient adherence to immunosuppressive medication as none adherence were remarkably in younger age as age less than 20 years. In addition to Pinsky, et al., (2009), who stated that adherence increase by age, none adherence noticed by adolescent and age of 19-24 and level increased dramatically at age of 25-44 years.

Ass for educational level this was consistent with Prihodova, et al., (2014), who mentioned that level of education had no significant effect on renal transplant recipients' adherence toward immunosuppressive medication but they found that patient who had a high level of education was fully adherent. This finding contrary with Griva, et al., (2012) and Denhearynck et al., (2009), who stated that level of education had a positive or negative significance on patients' level of adherence, also they found that patient who had high level of education was adherent to immunosuppressive.

Regarding to residence, the result of present study was contrasted with Denhaeynck et al., (2007), who mentioned that country origin affect patient adherence as patient who lived in United Stat versus nonUnited States. Finally, as regard to monthly income the finding of the current study was consistent with Weng, et al., (2013), who found that there was no significant difference between patients' income and their adherence 
toward immunosuppressive medication. On the posit side Danovitch., (2012) mentioned that low socioeconomic state is strong prophet of none adherence post renal transplantation. As well Prendgast, et al., (2010) found that none adherence was result of financial distress and it had a direct relationship between loss insurance, low income and graft failure. These differences were due Urology and Nephrology Center affiliated to Mansoura University offer immune-suppressive medication for free irrespective to economical status of renal transplant recipients.

Concerning association between renal transplant recipients' socio-demographic and economic characteristics and their total compliance score.

The finding of present study revealed that there was statistically significant association between age, and marital status of renal transplant recipients, and their total compliance score. Related to age the result of present study was contrasted with Omar, (2013), who mentioned that age had a significant effect on renal transplant recipients' compliance toward therapeutic regimen. It also agreement with Folkmane, et al., (2008), who mentioned that there was a negative association between patients' compliance and their age as older patient are most likely to be a good compliance and comply with recommendations. Concerned with marital status the result of present study was contrast with Denhaerynch, et al., (2009), who mentioned that none compliance was associated with patients who living alone, being unmarried or perceiving low social support. This was contrasted with Elsaadeny, et al., (2008), who mentioned that marital status had no significant difference on post renal transplant recipients level of compliance.

Concerning correlation between post renal transplant recipients' total adherence score and their total compliance score The finding of present study illustrated significant correlation between renal transplant recipients' total adherence score, and their total compliance score. This was consistent with Tielin, et al., (2014), they found that correlation with patients' compliance attitude and adherence to immunosuppressive medication.

\section{Conclusion}

The majority of renal transplant recipients had a good adherence to immunosuppressive medication and partial level compliancerelated to; medication, dietary recommendation, fluid, body temperature, and daily weight, avoiding infection, follow up visits, social relation, work post transplantation, physical exercise, marital relation, marital relation for male, and female, precaution of skin cancer, precaution of female breast cancer, and smoking. There was significant correlation between renal transplant recipients' adherence to immunosuppressive medication and compliance to recommended lifestyle post renal transplantation

\section{Recommendations:}

- Encourage periodical assessment for renal transplants' lifestyle, adherence to immunosuppressive medication, and compliance to therapeutic regimen.

- Develop and implement an educational program for renal transplant recipientsabout lifestyle modification.

- Identify patients whom were none adherent to medication or none compliant to recommended behavior pre transplantation and manage them.

- Further researches in different centers, and large sample to assess lifestyle modification, adherence to immunosuppressive medication, and compliance to therapeutic regimen to determine factors that may affect them.

\section{References}

[1]. . Abraham G., Mathew M., Lesley N, Ramani G., et al (2014)Nutritional Assessment of Renal Transplant Recipients Using DEXA and Biochemical Parameters, Nutritional Disorders \& Therapy J Nutr Disorders http://dx.doi.org/10.4172/21610509.1000134http://dx.doi.org/10.4172/2161-0509.1000134

[2]. Allender JA \& Spradley BW. (2010). Community Health Nursing, Promoting and Protecting the Public Health. $6^{\text {th }}$ Ed. London: Lippincott Willams and Wilkins. pp. 690-1.

[3]. Amaral TL, et al. (2013). Integrative Review on The Role of Nurses Post Kidney Transplant. Federal University of Acre Brazil. vol. 19(3) pp. 553-8.

[4]. Anita P RN \& Dadi ding RN (2016) After your kidney transplant, patient leadership committee the renal Net work, INC, This resource was created by ESRD Network 9/10 Patient Leadership Committee

[5]. Baker M A., and Goneim M A(2005) living donor renal transplantation 1976-2003 the Mansoura Experiences, Saudi J kidney transplant; 16(4)Pp573-538.

[6]. Bakr M A (2014) kidney transplantation clinical practice $1^{\text {st }}$ ed, AL-Mansoura Egypt Pp $95-112$

[7]. Bautista L., Stolzmann K., Gangnon R., McEloy J., Becker B., and Remington P(2007) Trends in kidney transplantation rates and disparities Medical Association Journal;99(8)Pp923-932

[8]. Bellizzia V , Cupistib A , Capitaninic A., Calellaa P and D’Alessandrob C .,(2014) Physical Activity and Renal Transplantation DOI: 10.1159/000355799 Published online: July 29, 2014

[9]. Brazil. vol. 19(3) pp. 553-8.

[10]. Brian RW, Nicki RC \& Stuart HR. (2011). Davidson's Principles and Practice of Medicine. 21 ${ }^{\text {st }}$ Ed. British. pp. 496-5.

[11]. Burkhalter H, Wirz-Justice A, Cajochen C, Weaver TE, Steiger J, Fehr T, Venzin RM, De Geest S.(2013) Daytime sleepiness in renal transplant recipients is associated with immunosuppressive nonadherence: a cross-sectional, multi-center study. Daytime sleepiness in renal transplant recipients is associated with immunosuppressive non-adherence: a cross-sectional, multi-center study 
Published by John Wiley \& Sons Ltd Clin Transplant 2013 DOI: 10.1111/ctr.12279 Clinical Transplantation

[12]. Butler JA, Roderick P, Mullee M, Mason JC \& Peveler RC. (2004). Frequency and Impact of Non-adherence to Immunosuppressant after Renal Transplantation: A Systematic Review. Transplantation. pp. 77- 769.

[13]. Chapman .J.R (2013) The KDIGO , clinical practice guide line for the care of kidney transplant recipients. Transplantation 89(6)Pp644-645)

[14]. Chisholm M A., (2004) Identification of medication- adherence barriers and strategies to increase adherence in recipients of renal transplants. Manag Care Interface; 17Pp44-8

[15]. Chisholm-Burns MA, Spivey CA \& Wilks SE. (2009). Social Support and Immunosuppressant Therapy Adherence among Adult Renal Transplant Recipients. DOI: 10.1111/j. 1399-0012.2009.01060.x.John Wiley and Sons A/S.

[16]. Chitra. U and Sunith.K.P(2013) nutrational management of renal transplant patient Research Gate Dio:10.1016/ j.ijt. 2013.05.004 available 
[50]. National Kidney Foundation (2015) what patient need to know available from

[51]. nkfcer@kidney.org

[52]. National Kidney Foundation (2012) Nutrition and transplantation, Pp 2-18 available at: www.kidney.org

[53]. National Kidney foundation. (2014). Organ Donation and Transplantation Statistics. ( June) 21. 2014. web: www.kidney.org

[54]. National Kidney foundation. (2013). Organ Donation and Transplantation Statistics. ( June) 21. 2013. web: www.kidney.org

[55]. Neyhart .C.D(2009) patient question about transplantation :A resource guide, nepherology nursing journal ,36(3) Pp279-285

[56]. Nielens H, Lalaoui A, Squifflet JP, Pirson Y, Goffin E, Lejeune TM (2010): Increase of physical activity level after successful renal transplantation: a 5 year follow-up study. Nephrol Dial Transplant;16:134-140.

[57]. Nourbala.J.R, Nemti.E, Rostami.B, (2012) impact of cigarette smoking of kidney transplant recipients. A systematic review, Iran. J, kidney Dis vol 5(3)Pp141-8

[58]. Omer Y A., (2009) Evaluation of follow up care among post kidney transplantation client, community health nursing, Ain shams University, Egypt

[59]. Patel D \& Lee. J ,( 2012) Medical Management of the Kidney Transplant Recipient edited by Sandip Kapur, Cheguevara Afaneh and Meredith J. Aull, ISBN 978-953-51-0900-6, INTECH, New York, NY, USA

[60]. Pezeshiki. M.(2013) pregnancy after renal transplantation : points to consider, Oxford Journal medicine Vol 17 issue 5 Pp703-707

[61]. Pinsky B W, Takemoto S K, Lentine K L, Burroughs T E, Schnitzler M A \& Salvalaggio P R (2009) Transplant outcomes and economic costs associated with patient noncompliance to immunosuppression. Am J Transplant., 9 (11): 2597-2606. 10.1111/j.1600-6143.2009.02798.x.

[62]. Prendergast M B and Gaston R S (2010) Optimizing Medication Adherence: An Ongoing Opportunity To Improve Outcomes After Kidney Transplantation ,Clin J Am Soc Nephrol 5: 1305-1311, 2010. doi: 10.2215/CJN.07241009

[63]. Prihodova L., Nogyova I., Rosenberger J., Majernikova M., Roland R., Goothff J W., Vandijk J P., and Wiley J P( 2014) Adherence in patients in first year after kidney transplantation and its impact on graft loss and mortality :A cross - sectional and prospective study, pubMed, 70(12)287-83 Dio: 10.1111/jan.12447

[64]. Ramadan S., Ismail M., Attia H., and Taha A(2013) assessment of renal transplant recipient needs at urology and nephrology center Unpublished Master Thesis, Faculty of Nursing, Banha University, Pp79-87

[65]. Rumyantzeu G ., Korford J., Baird B., Chelamcharla M., Habib A., Wand B., Lin S and Shihab F (2006) Role of socioeconomic status in kidney transplant outcome, Clin JAM, soc Nephrol;1(2)Pp313-322

[66]. Schmidt R J\& Anantharaman P(2007 ) Sexual function in chronic kidney disease. Adv Chronic Kidney Dis.; 14 P p :119-125.

[67]. Silva.D.S, Neto.E.D, Elias.R.M, Nahas.W.C , et al (2012) the perception of sleep quality in kidney transplant patient during the first year of transplantation, clinical science, CAPES, 67(12):Pp 1365-1371, Doi: 10.6061/ clinics/ 2012(12)04

[68]. Soltannezhad F, Fari Z, Morei M (2013) Effect of educating health promotion strategies on self- care self effecacy in patient undergoing kidney transplantation : a double Blind Randamized Trail, Nursing midwifery stud., 2(2):64-70 DOI: $10.5812 / \mathrm{nms} .11874$

[69]. Squifflet, J \& Paul. (2011). The History of Kidney Transplantation Past, Present and Future (with Special References to the Belgian History), Understanding the Complexities of kidney Transplantation. InJ. Ortiz \& J Andre (Ed), ISBN. pp. 307-891-9, 953-78- hptt: www. intechopen. com/books/.

[70]. Steddon S , Ashman N, Chesser A \& Cunningham J (2014) Oxford hand book of nepherlogy and hypertension $2^{\text {nd }}$ ed USA Pp: $308-334$

[71]. Tayebi A.,Raiesfar A., Ebadi A., Eynollahi B., Rafiyan Z., Keyvanloo F., (2010) investigation of renal transplantation patients quality of life by kidney transplantation questionnaire (KTQ25), Iranian journal of critical nursing vol 3 issue 3;Pp125-128.

[72]. Tielen M,1 Exel J , Laging M,1 Beck D K., , Khemai R, Gelder T, Betjes M, Weimar W, and.Massey E K(2014) Attitudes to Medication after Kidney Transplantation and Their Association with Medication Adherence and Graft Survival: A 2-Year FollowUp Study, Hindawi Publishing Corporation Journal of Transplantation Volume ID 675301, 9 pages http://dx.doi.org/10.1155/2014/675301

[73]. Trevit, R, Dunsmore V \& Murphy F, et al. (2012). Pre and Post Transplant Care Nursing Management of the Renal Transplant Recipient 38 (2) pp.107-14.

[74]. Urstad K H(2013) patient education for renal transplant recipients, Faculty of Medicine University of Oslo, Norway

[75]. Van der mai S F.,De jong P L., Groofthoff J W., Van de heuvel MJA., (2007) social participation after successful kidney transplantation, Disabil Rehabil,(29): 473-483.

[76]. Vanacker A and Maes B(2012). Malignancy Following Renal Transplantation, Chronic Kidney Disease and Renal Transplantation, ISBN: 978-953-51-0003-4, InTech, Available from: http://www.intechopen.com/books/chronic-kidney-disease-and-renaltransplantation/malignancy-followingrenal-transplantation

[77]. Vlaminck H, Maes B \& Evers G, et al. (2004). Prospective Study on Late Consequences of Sub Clinical Noncompliance with Immunosuppressive Therapy in Renal Transplant Patient. American Journal of Transplantation. vol . 4. No. 9 pp. 1509-13.

[78]. Weng F L., Chandwani Sh, Kurtyka K M., Zacker C, Marie A Chisholm B and Demissie K (2013) Open Access Prevalence and correlates of medication non-adherence among kidney transplant recipients more than 6 months post-transplant: a cross-sectional study, BMC Nephrology available at http://www.biomedcentral.com/1471-2369/14/26

[79]. World Health Organization (2012) renal transplantation available at http://www.who.int/bulletin/volumes/86/3/07041715len/accessed on 21 may 2012. 